\title{
Der Arzneimittelmarkt 2020 im Überblick
}

Carsten Telschow, Melanie Schröder, Jana Bauckmann, Katja Niepraschk-von Dollen und Anette Zawinell

\section{Inhaltsverzeichnis}

16.1 Die Entwicklung im Gesamtmarkt - 242

16.2 Wie verteilen sich die Umsätze und Verordnungen auf verschiedene Marktsegmente? - 245

16.2.1 Patentarzneimittel - 246

16.2.2 Marktdynamik der Arzneimittel bei seltenen Erkrankungen: Orphan-Arzneimittel - 255

16.2.3 Zweitanbieter: Der generikafähige und der biosimilarfähige Markt - 257

16.3 Wie verteilen sich die Nettokosten und Verordnungen auf die Pharmafirmen? - 263

16.4 Der Arzneimittelmarkt in der COVID-19-Pandemie - 266

$$
\text { Literatur - } 270
$$




\section{- Zusammenfassung}

Im Überblick zum Arzneimittelmarkt der gesetzlichen Krankenversicherung (GKV) im Jahr 2020 werden Ursachen und Hintergründe der Umsatzsteigerung um 4,9\% gegenüber 2019 analysiert. So wird mit Hilfe der Methode der Komponentenzerlegung die strukturelle Veränderung bei den Verordnungen als wichtigster Umsatztreiber für den durchschnittlichen Wert einer Arzneimittelverordnung identifiziert. Für diese Umsatzsteigerungen sind insbesondere neue und teure patentgeschützte Arzneimittel verantwortlich. Gleichzeitig sinkt der Anteil der Tagesdosen im Patentmarkt, sodass hier immer mehr Geld für immer weniger Versorgung aufzubringen ist. Dies trifft noch stärker für das stetig wachsende Marktsegment der Arzneimittel für seltene Erkrankungen (Orphan Drugs) zu, die mit extrem hohen Kosten jeweils nur bei wenigen Patient:innen angewendet werden und so einen Versorgungsanteil nach verordneten Tagesdosen von 0,06\%, aber einen Kostenanteil von 11,8\% am Gesamtmarkt ausmachen. Die Kosten im Zweitanbietermarkt bzw. Nicht-Patentmarkt stagnieren hingegen und auch die Erlöse aus Rabattverträgen haben 2020 das Niveau des Vorjahres erreicht. Viele der weltweit grö $\beta$ ten Pharmakonzerne konnten erneut Umsatzsteigerungen und höhere Gewinne verbuchen; alleine die 21 größten unter ihnen deckten über $50 \%$ der Umsätze im deutschen Arzneimittelmarkt 2020 ab, wobei diese Konzerne ihren Umsatz in Deutschland zu $70 \%$ mit Patentarzneimitteln erzielen. Ein Blick auf besondere Entwicklungen in der Arzneimittelversorgung während der COVID-19-Pandemie ergänzt die Sicht auf den GKV-Arzneimittelmarkt 2020.

\subsection{Die Entwicklung im Gesamtmarkt}

Im Jahr 2020 lagen die gesamten Ausgaben der GKV bei 267,8 Mrd. $€$ und damit um 8,9\% über den Ausgaben des Vorjahres (Amtliche Ausgabenstatistik KV 45). Zu den größ- ten Ausgabenposten gehören Krankenhausbehandlungen mit einem Anteil von 30,8\%, gefolgt von den Arzneimitteln mit einem Anteil von $17,1 \%$. Insgesamt summieren sich die Ausgaben für alle im Rahmen der GKV verordneten Produkte aus Apotheken wie Arzneimittel und Impfstoffe auf 47,8 Mrd. $€$ und sind gegenüber dem Vorjahr $(+5,9 \%)$ ähnlich stark angestiegen wie 2019 gegenüber 2018 $(+6,4 \%)$ (Bundesministerium für Gesundheit 2021).

Ausgehend von diesen Ausgaben ermittelt der GKV-Arzneimittelindex im Wissenschaftlichen Institut der AOK (WIdO) den Bruttoumsatz und die Nettokosten des GKVArzneimittelmarktes. ${ }^{1}$ Der Bruttoumsatz ist im Jahr 2020 gegenüber dem Vorjahr insgesamt um 4,9\% (2,3 Mrd. €) auf 49,2 Mrd. € angestiegen, was erneut ein deutliches Wachstum wie in den Vorjahren darstellt (• Abb. 16.1). Dieser Anstieg ergibt sich trotz einer sinkenden Verordnungsmenge (684 Mio. gegenüber 695 Mio. Verordnungen im vorherigen Jahr). Betrachtet man für einen längeren Zeitraum von zehn Jahren die Veränderungen gegenüber dem Jahr 2011, ergibt sich ein Anstieg des Bruttoumsatzes um $65 \%$ (19,5 Mrd. $€)$, jedoch nur ein Verordnungsanstieg um 9,4\% (58,7 Mio. Verordnungen).

\section{- Welche Komponenten beeinflussen den Umsatzzuwachs?}

Um die Ursachen des Umsatzanstiegs zu erklären, bietet sich das etablierte Konzept der Komponentenzerlegung ${ }^{2}$ der Umsatzent-

1 Die Marktanalysen des GKV-Arzneimittelindex betrachten Bruttoumsätze bzw. Nettokosten. In beiden Werten werden weder Ausgaben für Sprechstundenbedarf noch weitere Verordnungspositionen wie beispielsweise Verbandstoffe oder Teststreifen berücksichtigt. Zusätzlich sind darin die Zuzahlungen der Patient:innen enthalten. Ausgehend von den Bruttoumsätzen werden für die Nettokosten die gesetzlichen Abschläge für Hersteller und Apotheken abgezogen (WIdO 2021).

2 Eine detailliertere Beschreibung und Darstellung der Ergebnisse der Komponentenzerlegung der Umsatzentwicklung im Arzneimittelmarkt 2020 findet sich in WIdO (2021) 


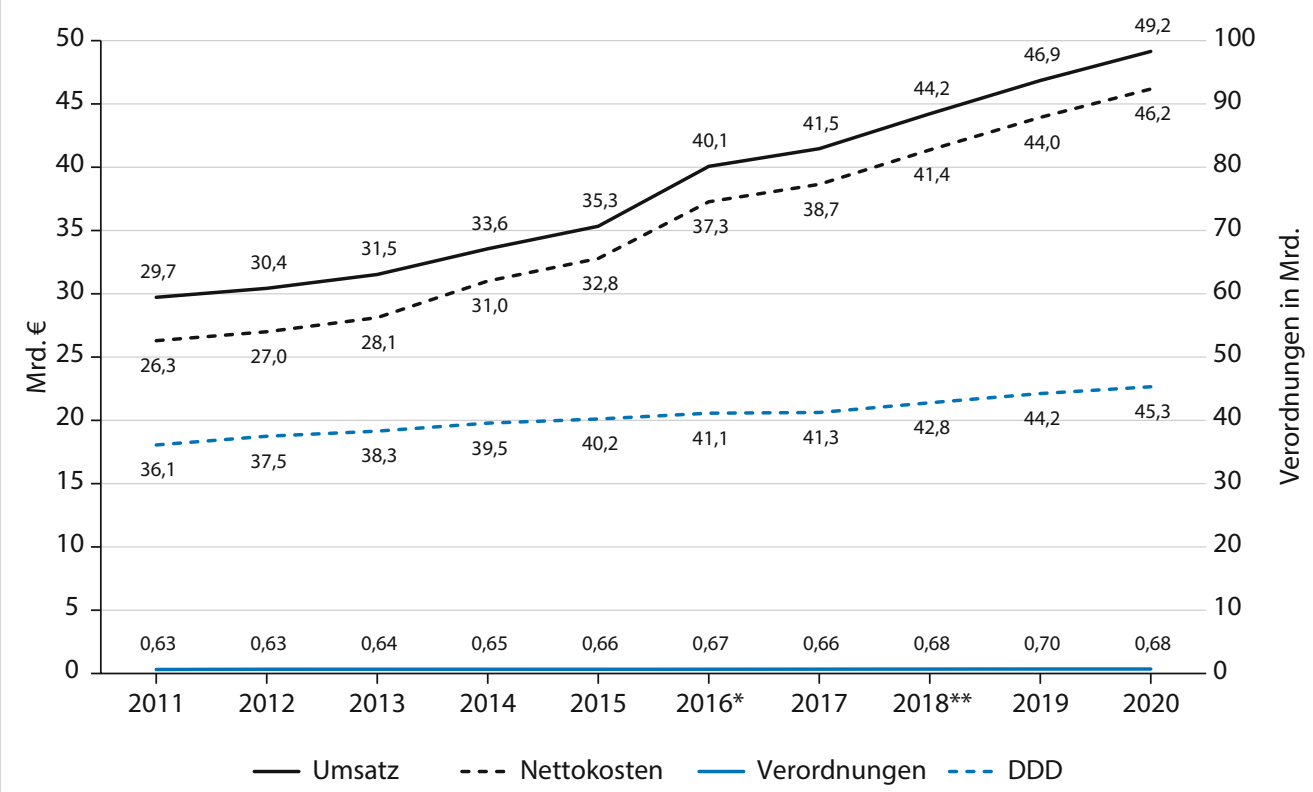

* Seit 2016 zusätzliche Berücksichtigung von parenteralen Zubereitungen

** Seit 2018 zusätzliche Berücksichtigung von ambulant abgegebenen Arzneimitteln aus Krankenhausapotheken

Arzneimittel-Kompass 2021

- Abb. 16.1 Bruttoumsatz, Nettokosten und Verordnungen des GKV-Arzneimittelmarktes seit 2011 (Quelle: GKVArzneimittelindex)

wicklung (Reichelt 1988; WIdO 2021) an, indem die Effekte einzelner Einflussfaktoren (sog. Komponenten) offengelegt werden. - Abb. 16.2 zeigt den Einfluss der einzelnen Faktoren auf die Umsatzveränderung $2020 \mathrm{zu}$ 2019.

Wie bereits zu $\bullet$ Abb. 16.1 beschrieben, zeigt sich auch in der Komponentenzerlegung, dass die Umsatzsteigerung um $4,9 \%$ bei einem negativen Mengeneffekt (Anzahl der Verordnungen: $-1,6 \%$ ) durch einen gestiegenen Wert je Verordnung hervorgerufen wird. So fielen für eine Verordnung im Jahr 2020 durchschnittlich 71,85€ Umsatz an, während es im Vorjahr noch $67,41 €$ waren - das entspricht einem Anstieg um 6,6\%. Beruht der Umsatzanstieg also darauf, dass Arzneimittel einfach nur teurer geworden sind?

Dem gestiegenen Wert je Verordnung steht ein deutlich negativer Preiseffekt gegenüber $(-2,3 \%)$, der den Effekt eines allgemeinen
Preisrückgangs der Produkte beschreibt, die sowohl 2019 als auch 2020 im Handel waren. Ursächlich für diesen Rückgang können unter anderem gesetzliche Maßnahmen zur Preisregulierung sein: So stellt beispielsweise das Preismoratorium sicher, dass Hersteller die Preise lediglich im Rahmen eines Inflationsausgleichs erhöhen können; gleichzeitig sorgen Festbetragsanpassungen und die nach dem Arzneimittelmarktneuordnungsgesetz (AMNOG) vereinbarten Erstattungsbeträge dafür, dass für viele Arzneimittel die Preise sogar abgesenkt wurden. Im Durchschnitt werden Arzneimittel, die auf dem Markt sind, im Laufe der Zeit preiswerter. Im Jahr 2020 kam ein weiterer Effekt hinzu: Die in der Pandemie im zweiten Halbjahr 2020 eingeführte vorübergehende Absenkung der Mehrwertsteuer von $19 \%$ auf $16 \%$ galt auch für Arzneimittel und deren Preise - dementsprechend gingen auch die Umsätze zurück. Ohne diese Maßnah- 


\begin{tabular}{|c|c|c|}
\hline Umsatzentwicklung & $4,9 \%$ & \\
\hline $\begin{array}{l}\text { Zahl der } \\
\text { Verordnungen }\end{array}$ & $-1,6 \%$ & \\
\hline Wert je Verordnung & $6,6 \%$ & \\
\hline Preis & $-2,3 \%$ & \\
\hline Warenkorb & $-0,5 \%$ & 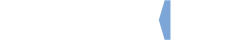 \\
\hline Struktur & $9,7 \%$ & \\
\hline Intermedikamenten & $8,2 \%$ & \\
\hline Intramedikamenten & $1,3 \%$ & D \\
\hline Darr./Stärken & $0,7 \%$ & ) \\
\hline \multirow[t]{2}{*}{ Packungsgrößen } & $0,7 \%$ & ) \\
\hline & & Arzneimittel-Kompass 2021 \\
\hline
\end{tabular}

- Abb. 16.2 Komponentenzerlegung der Umsatzentwicklung 2020 zu 2019 (Quelle: GKV-Arzneimittelindex)

me wäre das gesamte Umsatzwachstum mit $6,3 \%$ statt $4,9 \%$ noch deutlich höher ausgefallen.

Wie passen diese insgesamt sinkenden Preise aber $\mathrm{zu}$ einem steigenden und umsatztreibenden Wert je Verordnung? So wirken sich strukturelle Verschiebungen auch auf den Umsatz aus, weil sich in bestimmten Bereichen einige Arzneimittel immer stärker im Markt durchsetzen. Diese Effekte werden über die Intermedikamentenkomponente abgebildet. Bereits seit mehreren Jahren steigt der Intermedikamenteneffekt vergleichsweise deutlich an und ist sogar der Haupttreiber der Umsatzentwicklung. Eine bedeutende Ursache dafür ist die Veränderung der Therapie durch neue, aber auch teurere Arzneimittel. Bei vielen Erkrankungen sind große Therapiefortschritte erkennbar - es gibt mittlerweile erheblich besser wirksame Mittel gegen Infektionskrankheiten wie Hepatitis C und HIV, gegen Krebserkrankungen oder Autoimmunerkrankungen wie Rheumatoide Arthritis, chronisch entzündliche Darmerkrankungen oder Multiple Sklerose. Hintergründe, in welchen Bereichen sich diese strukturellen Verschiebungen und Teuerungen hauptsächlich abspie- len, zeigen die Kap. 5 Schröder und Telschow, $>$ Kap. 6 Ludwig und Vokinger sowie - Kap. 8 Haas et al. in dieser Publikation. Detaillierte Übersichten über die Ergebnisse der Komponentenzerlegung nach Wirkstoffgruppen (2. Ebene der Anatomisch-TherapeutischChemischen Arzneimittelklassifikation (ATCKlassifikation)) machen die verschiedene Effekte transparent (WIdO 2021).

\section{- Wie verteilen sich Umsätze und Verordnungen auf die Hauptindikationsgruppen?}

- Abb. 16.3 zeigt, wie sich ein Großteil der Nettokosten der GKV (rund 88\%) auf die zehn nettokostenstärksten der insgesamt 18 Hauptindikationsgruppen ${ }^{3}$ verteilt. Mehr als die Hälfte der Nettokosten entfällt allein auf nur drei Gruppen. Demnach gibt die GKV am meisten, nämlich 20,0\% der Gesamtnettokosten, für medikamentöse Krebstherapien aus. Diese stellen allerdings lediglich 0,6\% der gesamten verordneten Tagesdosen (Defined Daily Doses, DDD) dar. Ähnlich verhält es sich mit Immuntherapeutika: Auch diese Arzneimittelgruppe weist mit $17,3 \%$ einen hohen Nettokostenanteil auf, während ihr Verordnungsanteil mit 2,1\% der DDD vergleichsweise gering ist. Erst an dritter Stelle stehen Arzneimittel gegen Herz-Kreislauf-Erkrankungen, mit denen weitverbreitete chronische Krankheiten wie Bluthochdruck oder Koronare Herzerkrankung behandelt werden. Hier gibt die GKV rund sieben Mrd. $€$ $(15,6 \%)$ aus und deckt damit rund $50 \%$ des gesamten Verordnungsgeschehens ab.

Betrachtet man in der Abbildung die Anteilswerte vor zehn Jahren, so lassen sich insbesondere bei den Krebserkrankungen deutliche Veränderungen erkennen. Statt eines aktuellen Kostenanteils von 20,0\% lag dieser vor zehn Jahren mit nur 5,7\% noch auf deutlich niedrigerem Niveau und auf Ranglistenplatz sieben. Der Versorgungsanteil nach Tagesdo-

3 Zur Definition und Zuordnung der Hauptindikationsgruppen siehe WIdO (2021). 


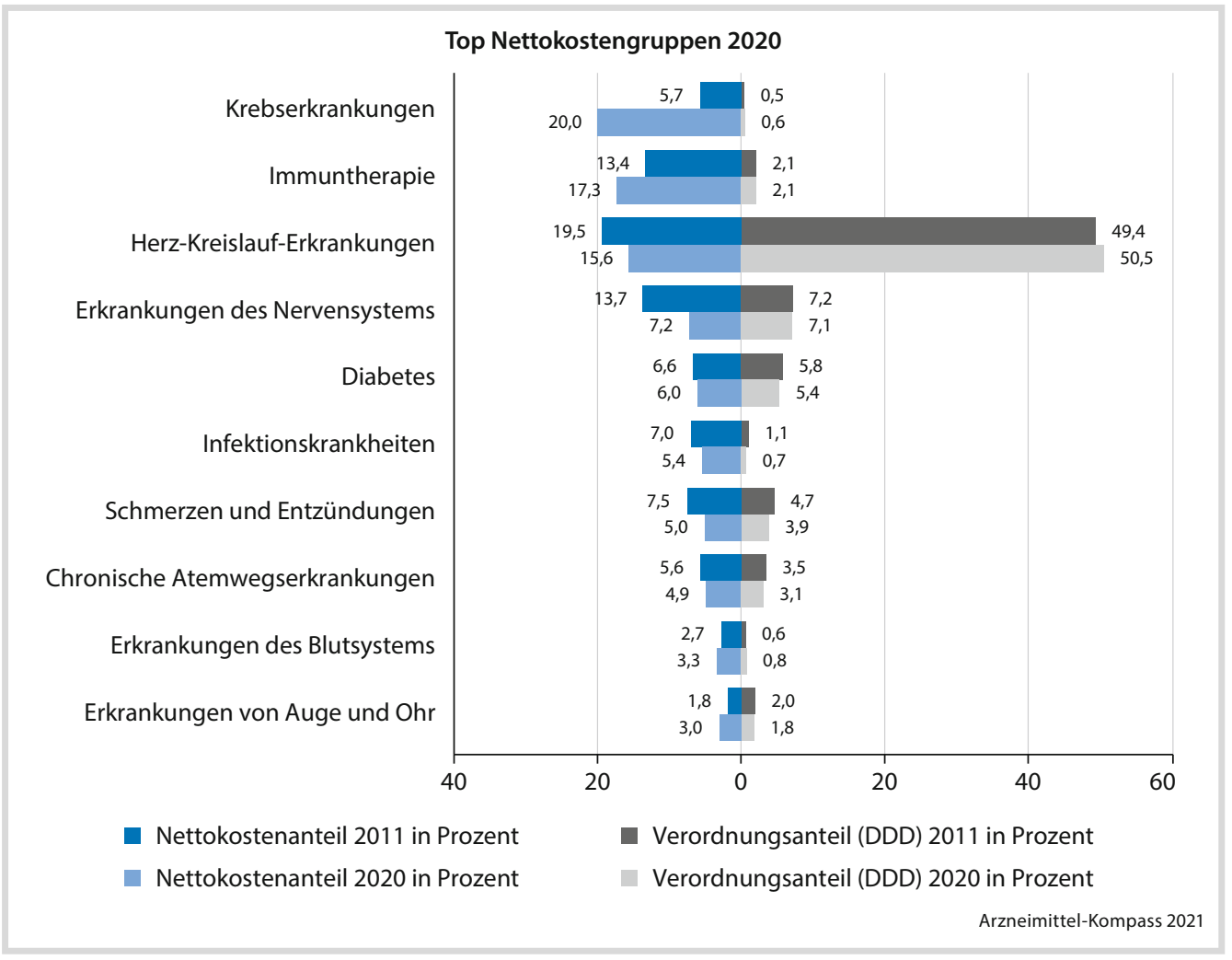

- Abb. 16.3 Die nettokostenstärksten Indikationsgruppen des Jahres 2020 im Vergleich ihrer Anteile der Nettokosten und Tagesdosen am Gesamtmarkt 2020 und 2011 (Quelle: GKV-Arzneimittelindex)

sen lag jedoch auch damals mit 0,5\% nur unwesentlich unter dem aktuellen Wert. In der Immuntherapie ist ebenfalls der Nettokostenanteil spürbar gestiegen - bei konstantem Versorgungsanteil. Der hohe Kostenanteil der Erkrankungen des Nervensystems vor zehn Jahren von $13,7 \%$ ist auch wegen zahlreicher Patentausläufe auf aktuell noch 7,2\% deutlich zurückgegangen; der Versorgungsanteil blieb dabei nahezu konstant. Weitere differenzierte Analysen werden mit dem PharMaAnalyst des WIdO ermöglicht. ${ }^{4}$

4 Der PharMaAnalyst des WIdO ist kostenfrei erreichbar unter https://arzneimittel.wido.de/ PharMaAnalyst.

\subsection{Wie verteilen sich die Umsätze und Verordnungen auf verschiedene Marktsegmente?}

Der Arzneimittelmarkt kann in verschiedenen Segmenten und Dimensionen dargestellt werden. In - Tab. 16.1 wird die Bedeutung der Marktsegmente Patentarzneimittel, Biologika und Orphan-Arzneimittel nach Nettokosten und den verordneten Tagesdosen dargestellt. 
Tab. 16.1 Nettokosten und verordnete Tagesdosen (DDD) sowie deren Anteile im GKV-Arzneimittelmarkt 2020 nach Marktsegmenten und Veränderungen zum Jahr 2019

\begin{tabular}{|c|c|c|c|c|c|c|c|}
\hline & $\begin{array}{l}\text { Netto- } \\
\text { kosten in } \\
\text { Mrd. } €\end{array}$ & $\begin{array}{l}\text { Verän- } \\
\text { derung } \\
\text { in \% }\end{array}$ & $\begin{array}{l}\text { DDD in } \\
\text { Mrd. }\end{array}$ & $\begin{array}{l}\text { Verän- } \\
\text { derung } \\
\text { in \% }\end{array}$ & $\begin{array}{l}\text { DDD- } \\
\text { Netto- } \\
\text { kosten } \\
\text { in } €\end{array}$ & $\begin{array}{l}\text { Netto- } \\
\text { kosten an } \\
\text { Gesamt } \\
\text { in \% }\end{array}$ & $\begin{array}{l}\text { DDD an } \\
\text { Gesamt } \\
\text { in \% }\end{array}$ \\
\hline Gesamtmarkt & 46,2 & 5,1 & 45,3 & 2,4 & 1,02 & 100 & 100 \\
\hline Patentarzneimittel* & 23,1 & 10,5 & 2,9 & 0,4 & 8,00 & 49,9 & 6,4 \\
\hline Nicht-Patentarzneimittel* & 23,1 & 0,2 & 42,4 & 2,5 & 0,54 & 50,1 & 93,6 \\
\hline Biologika & 14,7 & 8,9 & 1,4 & 3,0 & 10,78 & 31,8 & 3,0 \\
\hline Nicht-Biologika & 31,5 & 3,4 & 44,0 & 2,4 & 0,72 & 68,2 & 97,0 \\
\hline Orphan-Arzneimittel & 5,4 & 22,4 & 0,0 & 15,9 & 193,17 & 11,8 & 0,1 \\
\hline Nicht-Orphan-Arzneimittel & 40,8 & 3,1 & 45,3 & 6,0 & 0,90 & 88,2 & 99,9 \\
\hline \multicolumn{8}{|c|}{$\begin{array}{l}\text { *Die Zuordnung erfolgt in monatlicher Abgrenzung: Laufen die Schutzfristen für einen Wirkstoff beispielsweise } \\
\text { im Juli } 2020 \text { aus, so zählen die Arzneimittel bis Juli } 2020 \text { zum Patentmarkt und danach zum Nicht-Patentmarkt. } \\
\text { (Quelle: GKV-Arzneimittelindex) }\end{array}$} \\
\hline
\end{tabular}

\subsubsection{Patentarzneimittel}

Aus Wettbewerbssicht ist es entscheidend, ob für ein Arzneimittel bzw. seinen Wirkstoff noch Patente oder weitere Schutzfristen gültig sind. Dann ist Wettbewerbern der Markteintritt mit dem gleichen Wirkstoff nicht möglich. Hersteller genießen in diesem Fall eine auf maximal 20 Jahre befristete Nutzungsexklusivität, welche durch ein „ergänzendes Schutzzertifikat“ sowie Unterlagenschutz um maximal fünf weitere Jahre verlängert werden kann. Vor dem Hintergrund eines Entwicklungszeitraums von etwa zehn Jahren zwischen Patentanmeldung und marktfähigem Produkt kann somit von einer durchschnittlich zehn- bis fünfzehnjährigen tatsächlichen Marktexklusivität ausgegangen werden (Schweitzer und Lu 2018).

In - Tab. 16.1 zeigt sich, dass der häufig diskutierte Patentmarkt kostenmäßig nahezu genauso groß ist wie der Markt der Arzneimittel, bei denen die Schutzfristen abgelaufen sind und somit Wettbewerb entstehen kann. Hinsichtlich der Menge an Verordnungen spielt der Patentmarkt jedoch nur eine untergeordnete Rolle.

Die Nettokosten für den Patentmarkt lagen 2020 bei 23,1 Mrd. $€$ und haben sich gegenüber 2011 beinahe verdoppelt. Die Verordnungen entwickelten sich gegenläufig und sanken im gleichen Zeitraum um fast die Hälfte (-Abb. 16.4). Folglich haben sich die durchschnittlichen DDD-Nettokosten 2020 gegenüber $2011 \mathrm{mehr}$ als verdreifacht. Während die gesetzlichen Krankenkassen für eine Tagesdosis eines patentgeschützten Arzneimittels 2011 noch durchschnittlich $2,47 €$ ausgegeben haben, sind es im Jahr 2020 bereits $8,00 €$. Im Durchschnitt sind die Tagesdosiskosten mehr als 14-mal so hoch wie die der Nicht-Patentarzneimittel (vgl. - Tab. 16.1). Im nicht patentgeschützten Marktsegment stiegen die DDD-Nettokosten im selben Zeitraum von $0,42 €$ um vergleichsweise moderate $30 \%$ auf $0,54 €$. Dies zeigt, dass die Ausgaben und Preise im Patentmarkt deutlich stärker steigen als im restlichen Markt und damit die Gesamtkostenentwicklung deutlich beeinflussen (siehe Kap. 6 Ludwig und Vokinger). 


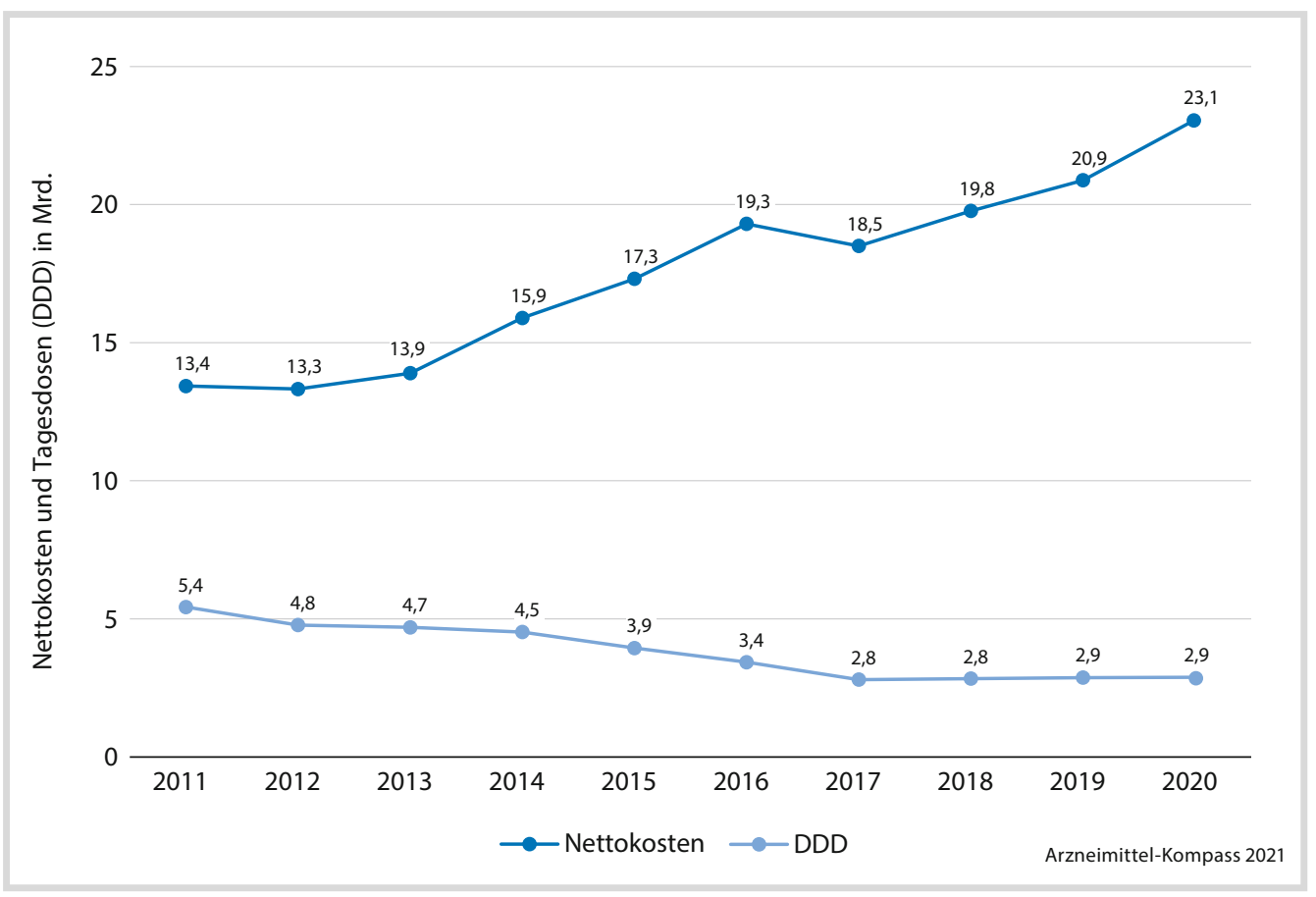

- Abb. 16.4 Verordnungen in Tagesdosen und Nettokosten im Patentmarkt seit 2011 (Quelle: GKV-Arzneimittelindex)

Dem Nettokostenanteil der patentgeschützten Arzneimittel von 49,9\% am Gesamtmarkt stand 2020 ein Verordnungsanteil nach Tagesdosen von lediglich $6,4 \%$ gegenüber. Vor zehn Jahren lag der Nettokostenanteil des Patentmarktes bei vergleichbaren $51,1 \%$ bei einem gleichzeitig noch deutlich höheren Anteil an verordneten DDD von $15,0 \%$ (• Abb. 16.5). Bereits seit Jahren nehmen demnach Verordnungen und Kosten insbesondere im Patentmarkt eine gegensätzliche Entwicklung. Immer mehr Kosten fallen für immer weniger Tagesdosen an.

Wie auch in $>$ Kap. 5 Schröder und Telschow gezeigt, sind es jedoch nicht pauschal die Arzneimittel des Patentmarktes, die immer teurer werden, sondern insbesondere die neuen Arzneimittel, die innerhalb der letzten 36 Monate auf den Markt gekommen sind und die häufig deutlich teurer sind als die bisherigen Therapiealternativen. Allein in den letzten zwei Jahren - im Zeitraum von Ja- nuar 2019 bis Dezember 2020 - hat sich mit Einführung mehrerer sehr teurer Präparate der durchschnittliche verordnungsungewichtete Packungspreis der Marktneueinführungen mehr als vervierfacht.

- Differenzierung im Patentmarkt: AMNOGArzneimittel und Bestandsmarkt

Mit dem Ziel, steigende Arzneimittelausgaben der gesetzlichen Krankenkassen zu reduzieren, ist am 1. Januar 2011 das Arzneimittelmarktneuordnungsgesetz (AMNOG) in Kraft getreten (Bundesministerium für Gesundheit 2016). Die damit eingeführte frühe Nutzenbewertung ( $\$ 35 \mathrm{a}$ des 5. Sozialgesetzbuchs, SGB V) brachte eine ordnungspolitische Richtungsänderung: Erstmals fanden eine transparente und evidenzbasierte Einordnung patentgeschützter Arzneimittel mit neuen Wirkstoffen in ihren therapeutischen Kontext und eine nachgelagerte Erstattungspreisvereinbarung zwischen dem pharmazeutischen Unternehmen und dem 


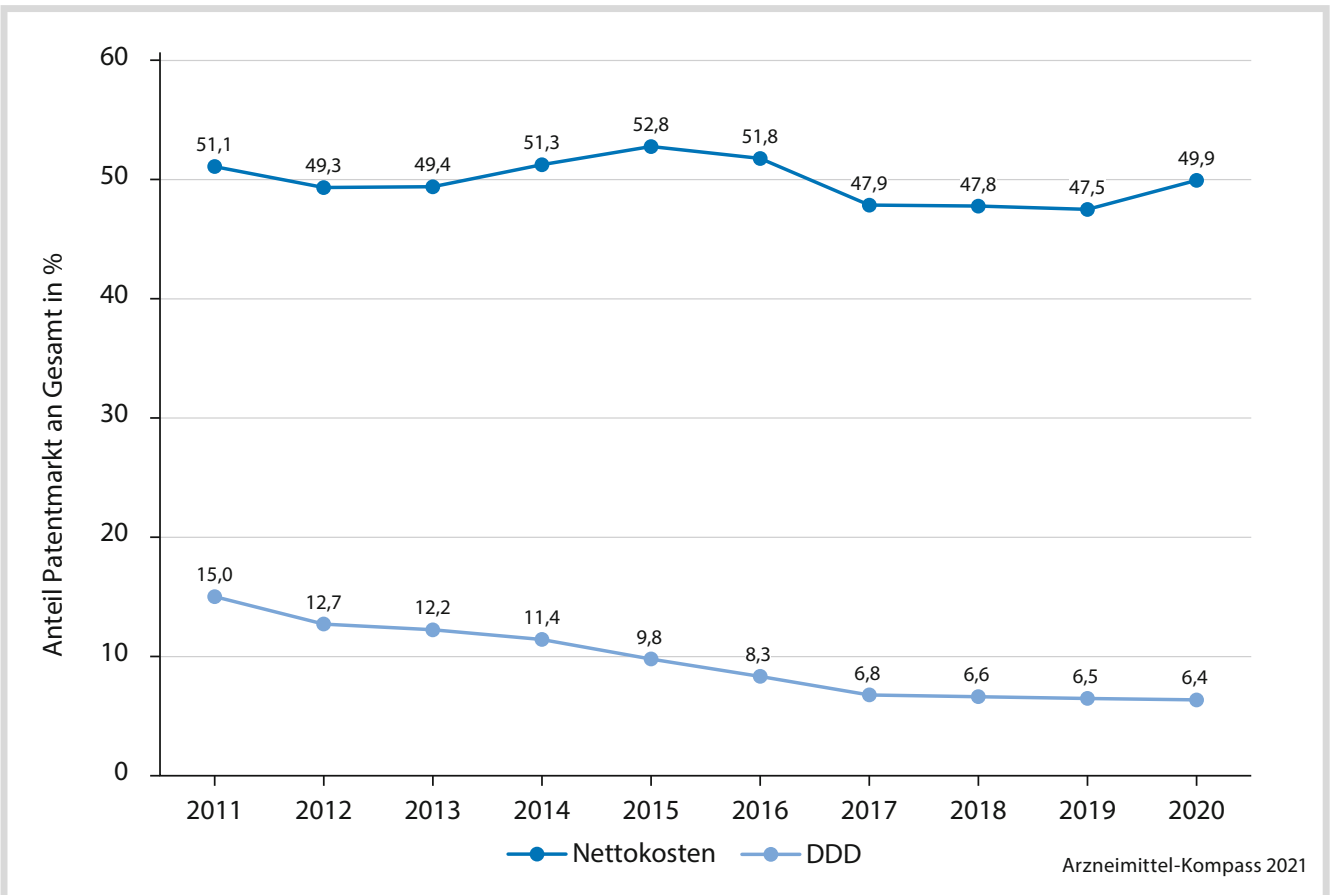

- Abb. 16.5 Verordnungsanteil nach Tagesdosen (DDD) und Nettokostenanteil des Patentmarktes seit 2011 (Quelle: GKV-Arzneimittelindex)

GKV-Spitzenverband statt. Vor 2011 oblag die Preisgestaltung allein dem pharmazeutischen Unternehmen. Mit dem AMNOG werden zwei maßgebliche Ziele verfolgt: Zum einen, Transparenz über den therapeutischen Stellenwert einer Arzneimittelinnovation herzustellen. Mit der abgeschlossenen Nutzenbewertung steht eine umfassende Bestandsaufnahme des aktuellen Informationsstandes für ein neues Arzneimittel zur Verfügung, die bei neuen Erkenntnissen - neue klinische Studien oder Auswertungen, Zulassungen für neue Anwendungsgebiete - regelhaft aktualisiert wird. Zum anderen wurde mit dem AMNOG auch erstmals die Möglichkeit geschaffen, die Preise neuer Arzneimittel kollektiv zu verhandeln und so wirksam die Arzneimittelausgaben zu dämpfen. Über die aktuellen Ergebnisse der Bewertungsverfahren und Preisverhandlungen gibt $>$ Kap. 17 Erdmann et al. Auskunft. Demgegenüber steht der Be- standsmarkt aus noch patentgeschützten Arzneimitteln, die mit ihrer Markteinführung vor 2011 noch nicht der Nutzenbewertung unterlagen.

Mit der schrittweisen Einführung neuer Arzneimittel auf der einen und dem Auslaufen von Patenten älterer Arzneimittel auf der anderen Seite verschieben sich die Anteile dieser zwei Gruppen innerhalb des Patentmarktes. Der Anteil der AMNOG-Arzneimittel steigt zwar seit 2011 sukzessive an, jedoch stellt der Bestandsmarkt immer noch ein großes Segment mit hohen Verordnungs- und Kostenanteilen dar. So hatte der Bestandsmarkt im Jahr 2020 noch immer einen Verordnungsanteil nach Tagesdosen von 58,2\% (1,73 Mrd. DDD) bzw. nach Nettokosten von 45,3\% (10,6 Mrd. €) innerhalb des gesamten Patentmarktes (• Abb. 16.6).

Ein Preismoratorium für die Arzneimittel des Bestandsmarktes, die nicht festbetragsge- 


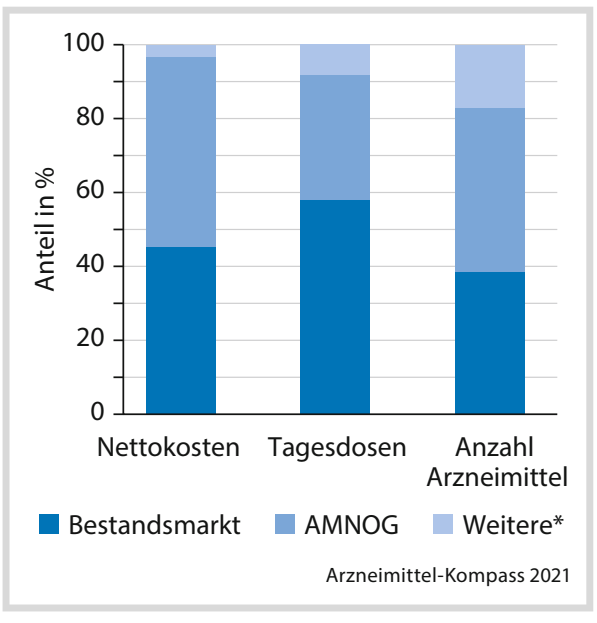

- Abb. 16.6 Anteile der AMNOG-bewerteten Arzneimittel und des Bestandsmarktes innerhalb des gesamten Patentmarktes 2020 nach Nettokosten, Tagesdosen sowie Anzahl der Arzneimittel (*Weitere Arzneimittel umfassen patentgeschützte Arzneimittel, die nach 2011 auf den Markt kamen, aber aus unterschiedlichen Gründen vom AMNOG nicht erfasst werden und keine Nutzenbewertungsverfahren durchlaufen.) (Quelle: GKV-Arzneimittelindex)

regelt sind, wird seit seiner Einführung 2009 bis heute aufrechterhalten und - nach wiederholter Evaluation - regelmäßig verlängert; aktuell gilt es noch bis Ende 2022. Damit können die Preise und Kosten dieser Arzneimittel begrenzt werden. Liegen die Herstellerabgabepreise über den Preisen zum Stichtag 01.08.2009, so muss der Hersteller die Differenz zwischen Stichtagspreis und Herstellerabgabepreis in Form von Herstellerabschlägen abführen (Regelung nach § 130a, Absatz 3a SGB V). Faktisch sind die Preise somit zum Stichtag eingefroren und können seit 2018 nur im Rahmen eines Inflationsausgleichs erhöht werden. Die meisten Hersteller nutzen inzwischen diese Möglichkeit für Preiserhöhungen. Die Kosten auf Basis der Herstellerpreise im Bestandsmarkt erhöhten sich dadurch im Jahr 2020 gegenüber 2019, wenn diese auch wegen der niedrigeren Inflationsrate geringer sind als in den Vorjahren. Als weiterer Effekt gehen auch die bisher zu leisten- den Preismoratoriumsabschläge zurück, da die wegen früherer Preiserhöhungen zu zahlenden Abschläge immer weiter abgeschmolzen werden. Zusammengenommen hat der Inflationsausgleich 2020 für meist ältere Produkte am Ende ihrer Marktexklusivität zu Mehreinnahmen der Hersteller in Höhe von 114 Mio. $€$ auf Ebene der Herstellerabgabepreise geführt. Wegen der Zuschläge der Handelsstufen und der Mehrwertsteuer entspricht dies im Jahr 2020 zusätzlichen Ausgaben für die GKV von 122 Mio. €.

\section{- Welche neuen Arzneimittel wurden im Jahr 2020 erstmals eingeführt?}

Die 31 neu in den ambulanten GKV-Markt eingeführten Arzneimittel des Jahres 2020 zeigt - Tab. 16.2. Darin ist der Wirkstoff Bempedoinsäure sowohl als Einzelwirkstoffpräparat als auch als Kombination mit einem weiteren Wirkstoff zweifach enthalten, sodass es sich um 30 neue Wirkstoffe handelt. Hier sind auch die Ergebnisse der Nutzenbewertung und die Differenzen zwischen dem ursprünglichen Listenpreis der Hersteller (Preis des pharmazeutischen Unternehmers (PPU)) und dem aktuell verhandelten Erstattungspreis (Abgabepreis des pharmazeutischen Unternehmers (APU)) gelistet. Auffällig ist, wie schon in den vergangenen Jahren, dass für eine Vielzahl von Präparaten als Ergebnis der Nutzenbewertung auf der Basis der zum Markteintritt verfügbaren Studienberichte keinen bzw. keinen belegbaren Zusatznutzen nachgewiesen werden konnte, diese aber verhältnismäßig häufig verordnet werden, wie - Tab. 16.2 zeigt. Auch wenn in der Vergangenheit gezeigt werden konnte, dass sich die mittlere Preissenkung $^{5}$ zwischen Arzneimitteln mit Zusatznutzen von denen ohne Zusatznutzen signifikant unterscheidet (Schröder und Telschow 2017; Schröder et al. 2020; Kap. 1 Greiner und Witte), stellt - Tab. 16.2 exemplarisch für die neuen Arzneimittel des Jahres 2020 dar, wie unterschiedlich diese Preisreduzierungen auch

5 Differenz zwischen ursprünglichem Listenpreis und verhandeltem Erstattungsbetrag. 


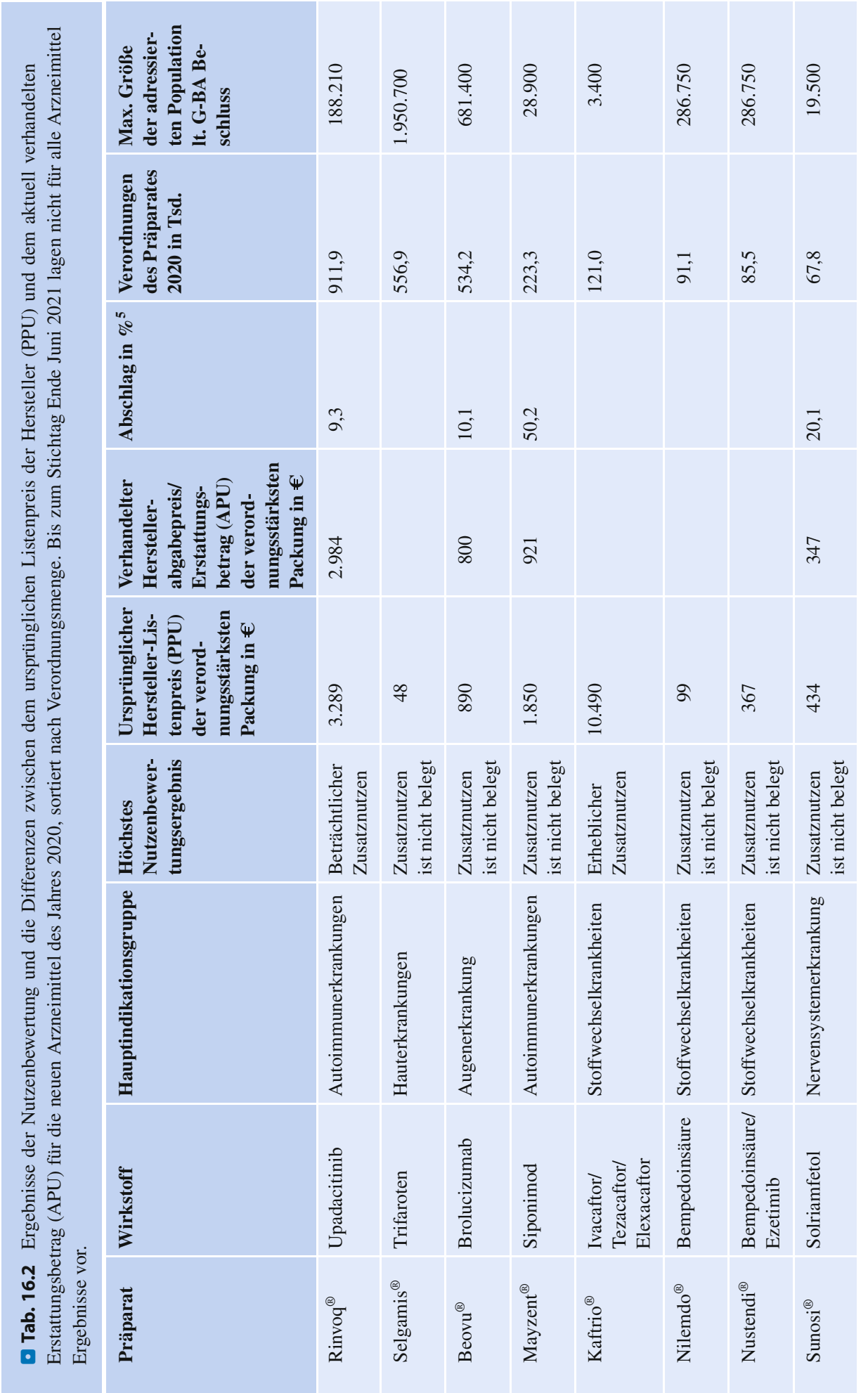




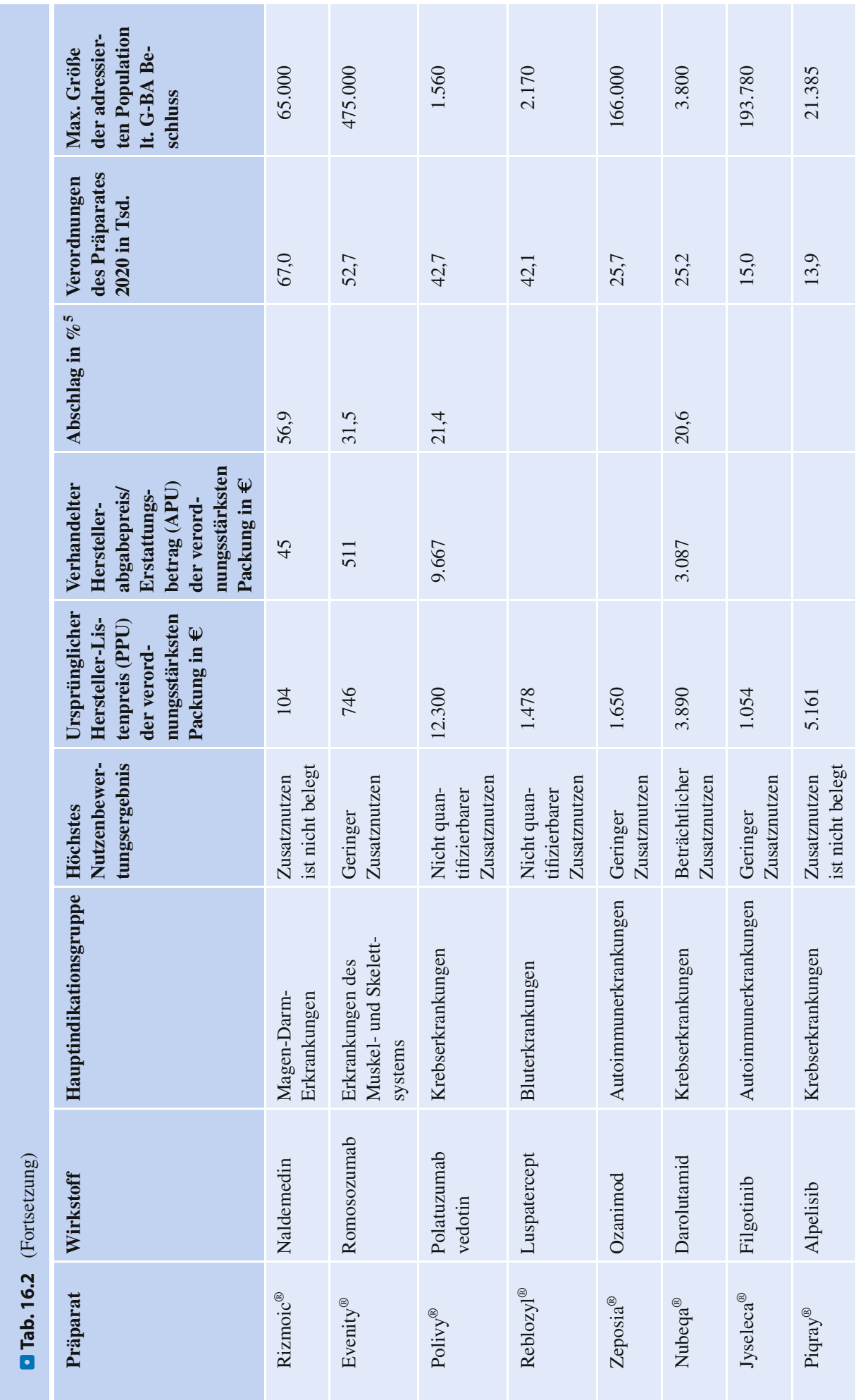



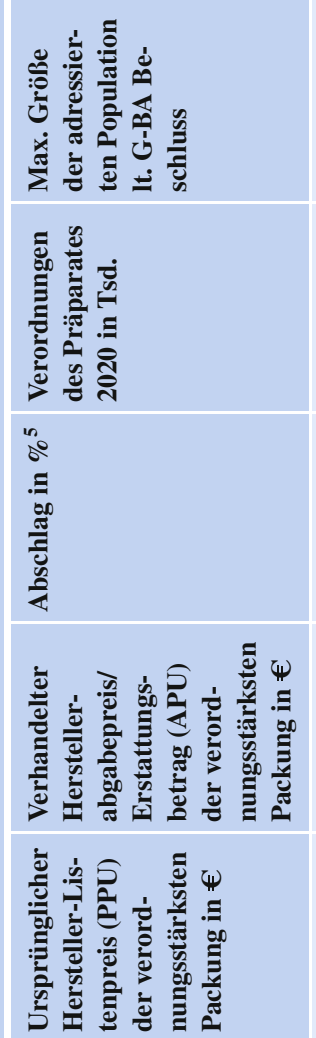

$\frac{8}{2} \stackrel{8}{a}$

oे $\stackrel{?}{=}$

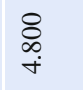

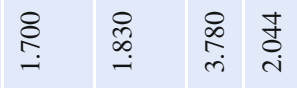

हैं

$\stackrel{8}{=}$

$\underset{\substack{n \\ \&}}{\stackrel{n}{n}}$

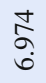
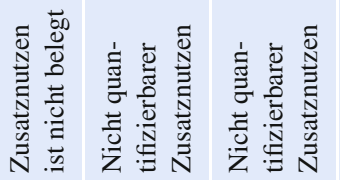

$\stackrel{\text { nิ }}{n}$

तु

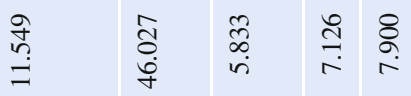

离

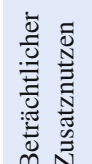
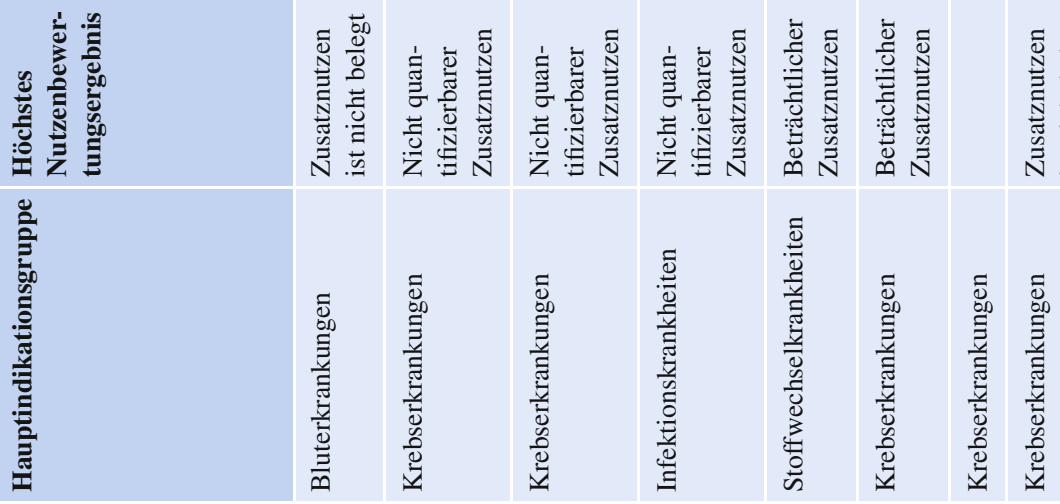

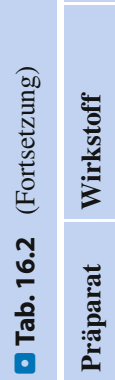

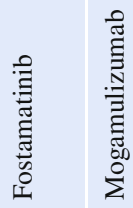

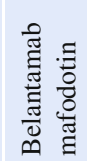

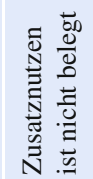


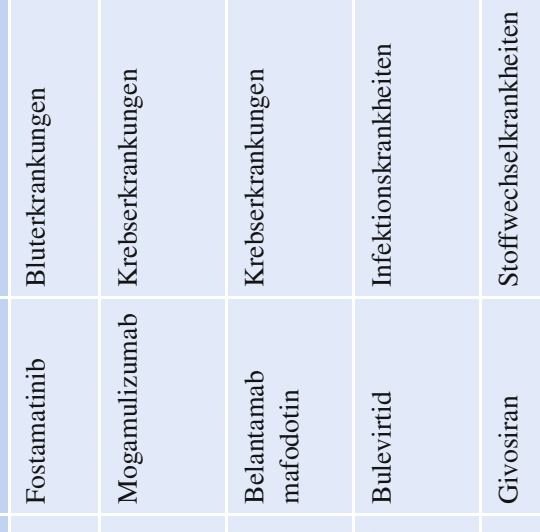

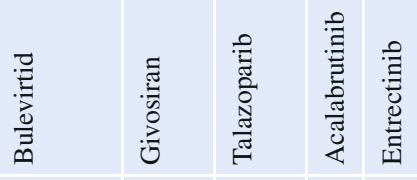

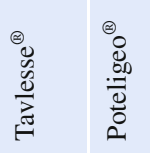

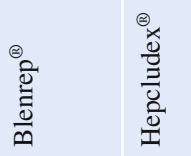

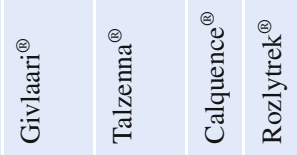



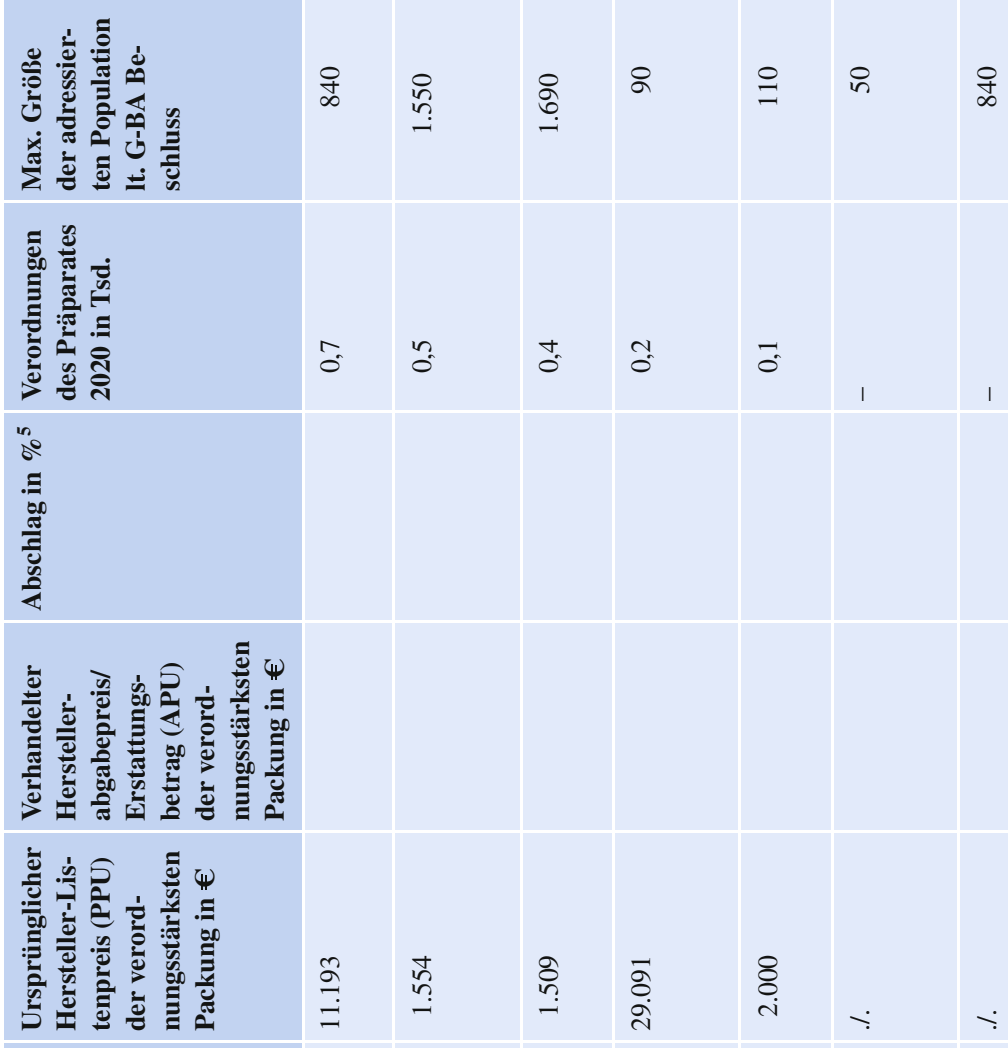

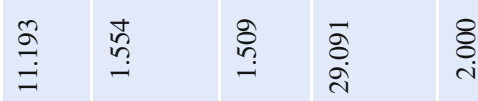
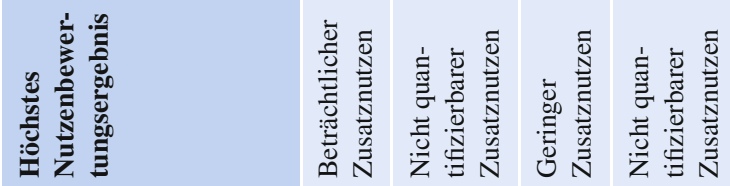

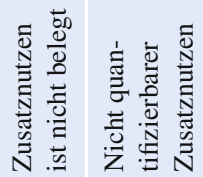
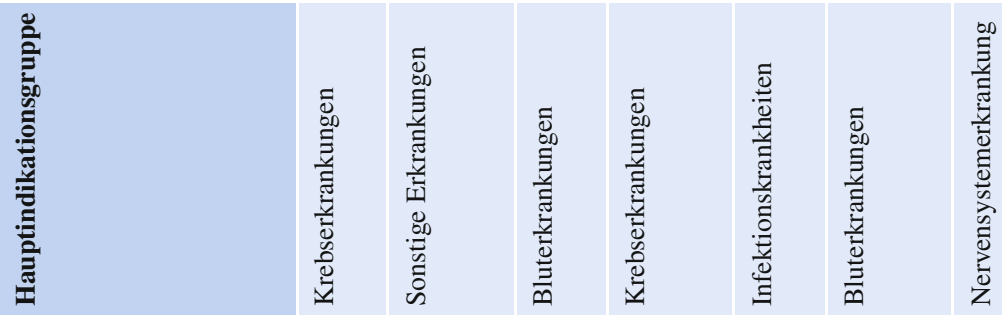

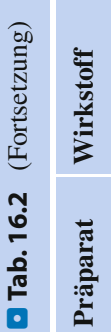

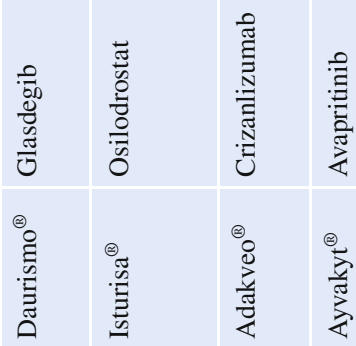
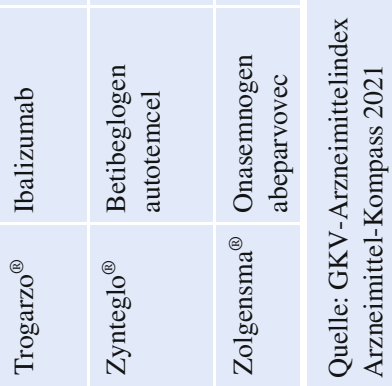
innerhalb einer Zusatznutzenkategorie ausfallen können.

Demnach erhalten die Arzneimittel des Jahres 2020 insgesamt einen Abschlag zwischen 9 und $57 \%$, während sich das Bild hinsichtlich des Zusatznutzens in der Tendenz nicht stark unterscheidet. Arzneimittel mit $\mathrm{Zu}$ satznutzen sind durch einen Abschlag zwischen 9 und $31 \%$ gekennzeichnet, während der Abschlag für Arzneimittel, denen kein $\mathrm{Zu}-$ satznutzen attestiert werden konnte, zwischen 10 und $57 \%$ liegt. Dabei ist zu bedenken, dass die Markteinführungspreise vom pharmazeutischen Hersteller frei gewählt werden und sich die Rationalität bei der Festlegung der Preise zwischen den pharmazeutischen Herstellern oder den verschiedenen Produkten deutlich unterscheiden dürfte. Auch die Anzahl der adressierten Patient:innen hat einen deutlichen Einfluss auf die Wahl und Einigung des Preises. Je kleiner die Population bzw. je spezieller oder spezifischer die Behandlung, desto höher ist meist der Preis. - Tab. 16.2 zeigt für einige Arzneimittel nur sehr geringe Patient:innenzahlen. Werden später mit erweiterten Zulassungen weitere Teilpopulationen adressiert, steigt die Wahrscheinlichkeit, dass die Gesamtkosten überproportional zunehmen, da das Verhältnis zwischen der Zahl der Patient:innen und dem Preis sich nicht entsprechend anpasst.

\section{- Welche finanziellen Auswirkungen ergeben sich für die neuen Arzneimittel des Jahres 2020?}

Stellt man die Umsätze der Arzneimittel, die in den Jahren 2011 bis 2020 einer frühen Nutzenbewertung unterzogen wurden und für die bis Ende 2020 ein Erstattungsbetrag verhandelt wurde, den Umsätzen gegenüber, die sich ergeben hätten, wenn der verhandelte Erstattungsbetrag bereits bei Markteinführung gegolten hätte, zeigen sich die Mehrbelastungen der GKV durch die herstellerseitige freie Preisbildung im ersten Jahr. Addiert man diese Mehrkosten, die aufgrund der freien Preisbildung im ersten Jahr angefallen sind, so ergeben sich in Summe 1.289 Mio. $€$, die die GKV in den Jahren 2011 bis 2020 hätte einsparen können, wenn die vereinbarten Erstattungsbeträge bereits ab der Markteinführung gültig gewesen wären. Allein für die neuen Arzneimittel des Jahres 2020 summieren sich diese Mehrkosten auf 46 Mio. $€$ mit den bisher verhandelten Erstattungsbeträgen, die jedoch zum Zeitpunkt der Berechnung erst für ein Drittel der neuen Arzneimittel bekannt waren. Umsätze von Festbetrags-Arzneimitteln und von Arzneimitteln ohne Erstattungsbeträge (wegen Marktrückzügen, fehlenden Preiskennzeichens oder noch fehlender Preiseinigung) sind in dieser Berechnung nicht enthalten, da für diese keine Preisdifferenzen berechnet werden können.

Doch nicht nur zur Markteinführung werden Erstattungsbeträge verhandelt. Erneute Preisdiskussionen schließen sich im Regelfall auch nach einer Neubewertung an, wenn beispielsweise ein neues Anwendungsgebiet zugelassen wird und damit größere Patientenpopulationen neu erschlossen werden oder wenn sich die Bewertung des Zusatznutzens ändert, beispielsweise bei Vorliegen neuer Studienergebnisse. Auch hier sollte es möglich sein, den verhandelten Erstattungsbetrag zwölf Monate rückwirkend gelten zu lassen. Denn die Patient:innen im neuen Anwendungsgebiet können schon ab dem Zeitpunkt der Zulassungserweiterung mit dem Arzneimittel behandelt werden, der neu verhandelte Preis steht aber erst ein weiteres Jahr später fest (Litsch et al. 2019). Bezieht man diese - sowohl positiven als auch negativen - Preisänderungen nach Neubewertungen in die Berechnung mit ein, so addieren sich die Mehrkosten seit 2011 sogar auf 2,02 Mrd. $€$ für zu hohe Preise aufgrund bislang nicht möglicher rückwirkender Geltung, davon allein 176 Mio. $€$ für das Jahr 2020. Die Hersteller profitieren also weiterhin deutlich von der freien Preisbildung, insbesondere solche, die für ihre Arzneimittel keinen überzeugenden Nutzen belegen konnten und infolgedessen höhere Preisabschläge nach dem ersten Jahr hinnehmen müssen. Entsprechende gesetzliche Anpassungen, die solche Effekte vermeiden helfen könnten, werden in - Kap. 12 Richard et al. beschrieben. 


\subsubsection{Marktdynamik der Arzneimittel bei seltenen Erkrankungen: Orphan- Arzneimittel}

Ein weiteres Segment des Arzneimittelmarktes, das sich durch besonders hohe Tagesdosiskosten auszeichnet, sind Arzneimittel, die zur Behandlung von seltenen Erkrankungen eingesetzt werden, die sogenannten Orphan-Arzneimittel. Dies wird daran deutlich, dass nur $0,06 \%$ der verordneten Tagesdosen einen Nettokostenanteil von $11,8 \%$ erreichen. Hier kostet eine Tagesdosis im Durchschnitt 193,17€ (- Tab. 16.1); mehr als das Zweihundertfache der Therapie mit Nicht-Orphan-Arzneimitteln. Zwar finden diese in der Regel jeweils nur bei wenigen Patientinnen und Patienten Anwendung, aber es kommen gleichzeitig immer häufiger Orphan-Arzneimittel für mehr seltene Erkrankungen in den Markt. So wurden allein in den vergangenen zehn Jahren 126 neue Orphan-Arzneimittel in den deutschen Markt eingeführt und waren zu den von den Herstellern gesetzten Preisen erstattungsfähig, was im europäischen Vergleich ungewöhnlich liberal erscheint, zumal diese speziellen Arzneimittel in ihrer Entwicklung und Marktpositionierung von zahlreichen regulativen und finanziellen Vorteilen profitieren (Überblick bei Schröder et al. 2020). Zusammengenommen haben die Orphan Drugs inzwischen mit 5,4 Mrd. $€$ und $11,8 \%$ der gesamten Nettokosten einen beträchtlichen Marktanteil erreicht. Orphan Drugs nehmen daher bei der Diskussion um zukünftige Marktregulierungen einen wichtigen Platz ein (Marselis und Hordijk 2020; Kap. 6 Ludwig und Vokinger sowie $\checkmark$ Kap. 8 Haas et al.).

Im Jahr 2020 war erneut ein hoher Anteil der neuen Wirkstoffe im deutschen Markt bei Zulassung in einem Arzneimittel gegen seltene Leiden enthalten (• Abb. 16.7). Davon entfällt fast die Hälfte der Wirkstoffe auf onkologische Indikationen. Dass die Entwicklung von Orphan-Arzneimitteln zur punktuell molekularen Behandlung onkologischer Erkrankungen eher im Mittelpunkt des Forschungsinteresses der pharmazeutischen Unternehmen steht und nicht die Entwicklung von Arzneimitteln gegen seltene genetisch bedingte Erkrankungen, für die es häufig keine Therapieoptionen gibt, wird dabei kritisch betrachtet (Ludwig 2019).

Infolgedessen nehmen auch die Nettokosten und - allerdings in deutlich geringerem Maße - die verordneten Tagesdosen der Arzneimittel zu, die jemals als Orphan Drug zugelassen wurden. Während Orphan-Arzneimittel 2011 noch Nettokosten in Höhe von ca. 1 Mrd. $€$ verursachten, was $4 \%$ der Nettokosten des gesamten GKV-Marktes entsprach, haben sich diese Kosten in zehn Jahren verfünffacht und erreichen mit über 5,4 Mrd. $€ 2020$ einen Nettokostenanteil von $12 \%$ (• Abb. 16.8). Allein der Vergleich von 2020 zu 2019 zeigt: Mit einem Nettokostenwachstum in Höhe von $22,4 \%$ ist die Nettokostenentwicklung in diesem Marktsegment deutlich dynamischer als im Restmarkt (3,1\%, - Tab. 16.1). Im Jahr 2020 wurden 108 aktuell klassifizierte Orphan Drugs verordnet. Rund $42 \%$ der Nettokosten der Orphan-Arzneimittel in Deutschland entfallen auf Krebserkrankungen. Lediglich bei rund $12 \%$ Nettokosten aller Orphan-Arzneimittel handelt es sich um Arzneimittel zur Enzymersatztherapie, die bei einer typischen schweren chronischen seltenen Erkrankung wie Morbus Pompe zur Anwendung kommen. Die Nettokosten für eine Tagesdosis unter den Orphan-Arzneimitteln rangieren zwischen 5 und $9.019 €$, im Durchschnitt liegen sie bei 193,17€ Nettokosten je Tagesdosis, während sie im Gesamtmarkt bei 1,02€ liegen und im Patentmarkt im Mittel bei $8,00 €$ (- Tab. 16.1).

Bis 2024 wird für den weltweiten Markt der Anteil der Orphan Drugs an den Gesamtausgaben auf $18 \%$ prognostiziert (EvaluatePharma 2020). Für rund $40 \%$ der Arzneimittel gegen seltene Erkrankungen, die sich in der Entwicklungspipeline befinden, wird 2024 der Status eines Blockbusters, also eines Arzneimittels, das jährlich mehr als 1 Mrd. US\$ Umsatz erzielt, erwartet (EvaluatePharma 2018). Allein im deutschen Markt hat das Arzneimit- 


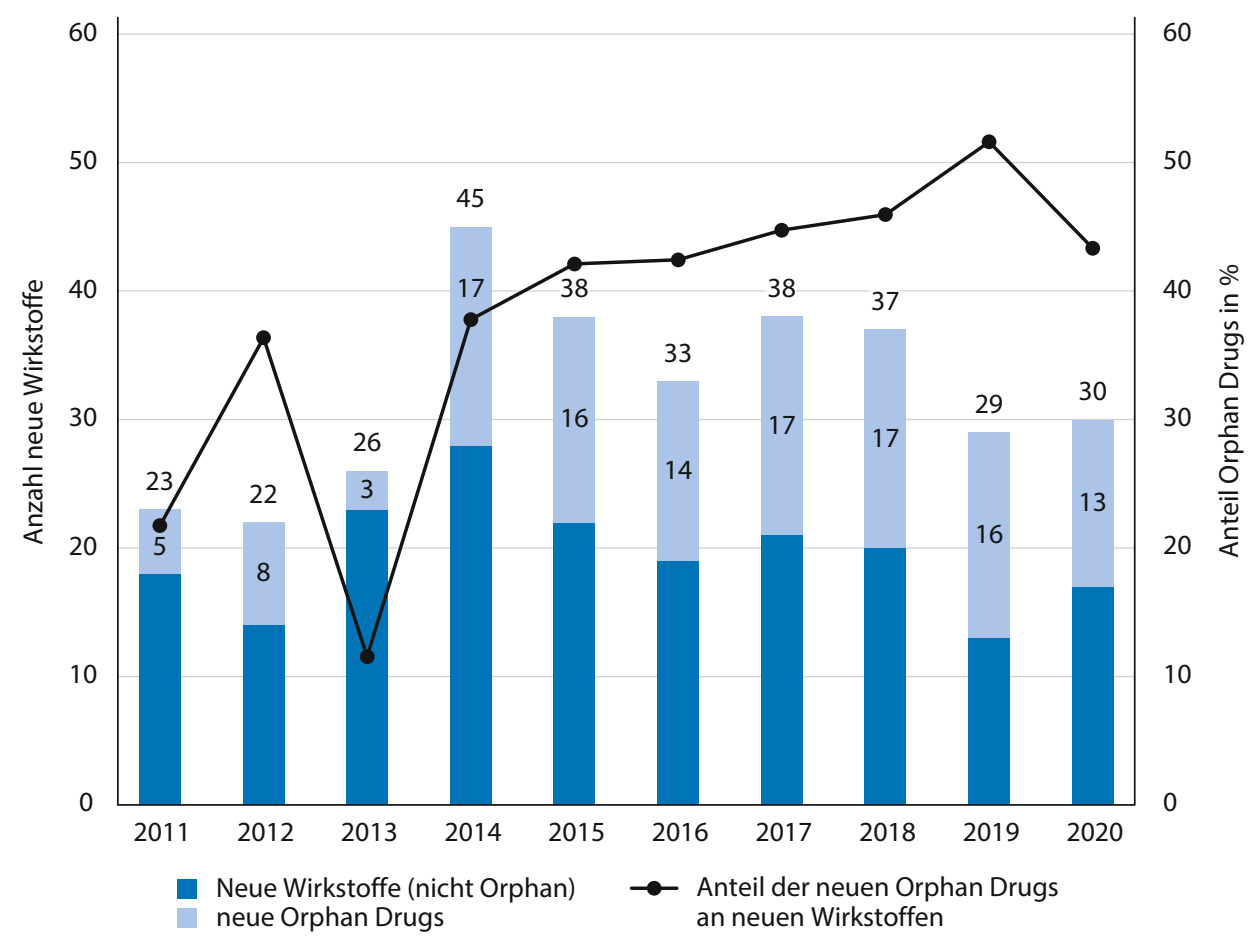

Arzneimittel-Kompass 2021

- Abb. 16.7 Gesamtanzahl der neu eingeführten Wirkstoffe seit 2011 und Anzahl und Anteil der neuen Arzneimittel, die bei Zulassung eine Orphan-Designation besaßen. (Quelle: GKV-Arzneimittelindex)

tel Revlimid ${ }^{\circledR}$ einen Umsatz von 746 Mio. $€$ erreicht. Zwar hatte es Ende 2019 seinen Orphan-Drug-Status verloren, jedoch bis dahin dem Hersteller alle mit diesem Status verbundenen Vorteile verschafft. Auch für das neuere Imbruvica ${ }^{\circledR}$ steht mit 405 Mio. $€$ Umsatz im Jahr 2020 zu erwarten, dass es ein Blockbuster wird. Doch wie passt zusammen, dass Arzneimittel gegen seltene Erkrankungen Blockbuster sind? Die bereits skizzierten Indikationsausweitungen auf weitere $\mathrm{Pa}$ tient:innengruppen bedingen, dass viele dieser Arzneimittel ihre Verordnungsmengen verviel- fachen können, der einmal gesetzte Preisanker jedoch meist nicht entsprechend gesenkt wird (vgl. auch Schröder et al. 2020). Ohnehin ist die Möglichkeit für Preissenkungen über Erstattungsbeträge begrenzt, da mit dem gesetzlich festgelegten Zusatznutzen für Orphan Drugs wenige Argumente für die Preisverhandlungen bleiben. Es mutet paradox an, dass mit den heutigen Bedingungen Arzneimittel in ihrer Entwicklung massiv gefördert werden, die dann mit dem Status als Orphan Drugs hohe Umsätze generieren, ja sogar zu Blockbustern werden. 


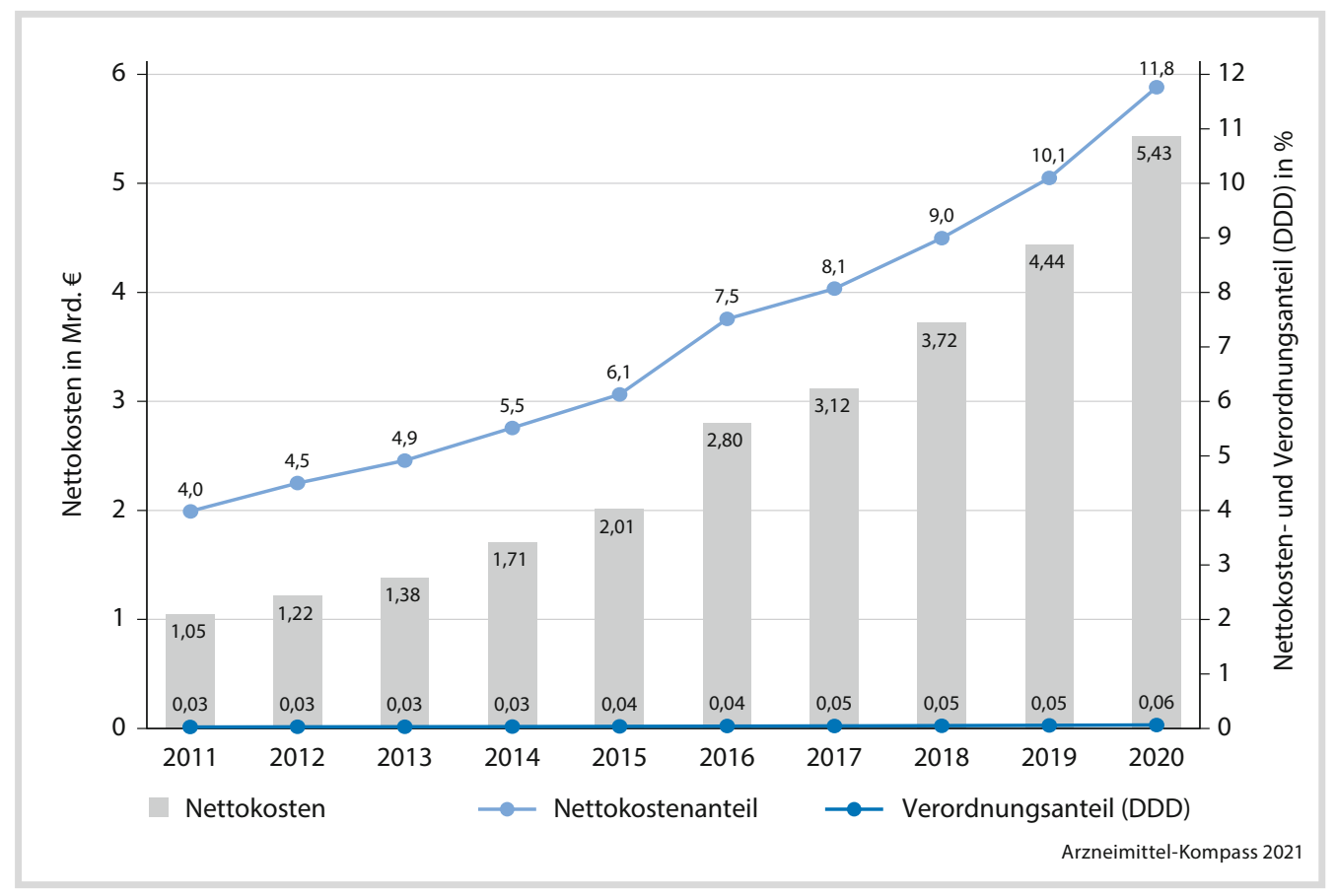

- Abb. 16.8 Entwicklung der Nettokosten sowie Nettokosten- und Verordnungsanteile (DDD) der Orphan Drugs seit 2011 (Quelle: GKV-Arzneimittelindex)

\subsubsection{Zweitanbieter: Der generikafähige und der biosimilarfähige Markt}

Der Zweitanbietermarkt entsteht nach Ablauf von Patenten und Schutzfristen, wenn weitere Anbieter mit wirkstoffgleichen Präparaten den Markt betreten können und so ein Wettbewerb entsteht. Der Zweitanbietermarkt lässt sich differenzierter hinsichtlich der Herstellungsart bzw. der Produkteigenschaften in den generikafähigen Markt - für chemisch-synthetisch hergestellte Wirkstoffe - und den biosimilarfähigen Markt - für bio- oder gentechnologisch hergestellte Wirkstoffe (Biologika) betrachten. Mit 20,65 Mrd. $€$ Nettokosten und 601,7 Mio. Verordnungen (40,2 Mrd. Tagesdosen) stellen die generikafähigen Wirkstoffe einschließlich ihrer jeweiligen Originale - den größten Teil des Zweitanbietermarktes im Jahr 2020. Der Wettbewerbsmarkt der Biologika, auf die im Jahr 2020 insgesamt 4,0 Mrd. $€$ und 593 Mio. DDD entfallen, hat durch Patentausläufe in den letzten Jahren deutlich zugenommen (• Abb. 16.9). Hier sind zusätzlich zu den Biosimilars und ihren Referenzprodukten auch weitere Originale vergleichbarer Wirkstoffe enthalten, die im direkten Wettbewerb zueinander stehen. Für die beispielsweise im Jahr 2020 in Summe trotz zwei neu hinzugekommener biosimilarfähiger Wirkstoffe in der zweiten Jahreshälfte abnehmenden Nettokosten gibt es mehrere Ursachen, die im Folgenden näher betrachtet werden.

In den letzten Jahren ist zu beobachten, dass die meist günstigeren Zweitanbieter-Biologika immer schneller den Markt durchdringen. So erzielte der erst seit 2019 biosimilarfähige Wirkstoff Adalimumab bereits nach 12 Monaten einen Marktanteil an Biosimilars von $54 \%$, während dies beim - in vergleichbaren Indikationen eingesetzten, aber bereits seit 2015 biosimilarfähigen - Infliximab erst nach 


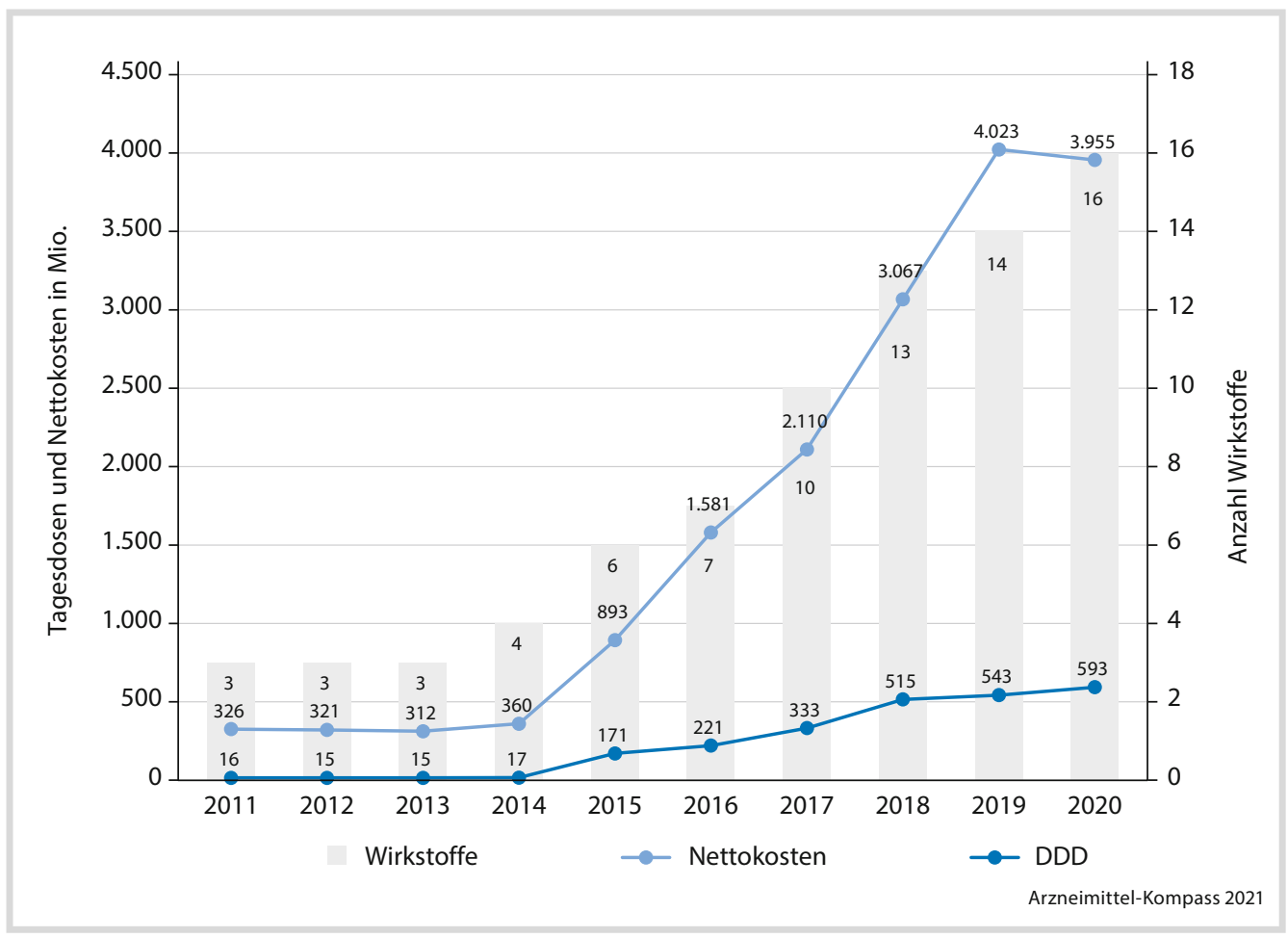

- Abb. 16.9 Nettokosten und Verordnungen (DDD) sowie Anzahl der biosimilarfähigen Wirkstoffe seit 2011 (Quelle: GKV-Arzneimittelindex)

33 Monaten erreicht wurde. Die Gründe hierfür sind vielfältig und offenbar auch wirkstoffabhängig: Die ebenfalls seit 2019 angebotenen Teriparatid-Biosimilars haben nach 12 Monaten erst $24 \%$ Marktanteil erreicht, während für das vorwiegend bei Krebserkrankungen eingesetzte Bevacizumab bereits im vierten Monat der 2020 begonnenen Patentfreiheit ein Biosimilar-Marktanteil von $69 \%$ erreicht wurde. Gerade für Wirkstoffe mit ehemals hohen Umsätzen bietet sich im Wettbewerb viel Potenzial für Kostensenkungen. Die Gegenüberstellung der Zweitanbieteranteile im biosimilarfähigen und generikafähigen Markt in • Abb. 16.10 zeigt, dass der generikafähige Markt mit deutlich stärker ausgeprägtem Wettbewerb insgesamt auch höhere Zweitanbieteranteile bei den Verordnungen aufweist, als dies für den meist noch immer überschaubaren Wettbewerb im biosimilarfähigen Markt festzustellen ist.
Insgesamt werden hierbei 503 generikafähige Wirkstoffe bzw. Wirkstoffkombinationen und 16 biosimilarfähige Wirkstoffe berücksichtigt, die im Jahr 2020 verordnet wurden.

Auch bei den durchschnittlichen Preisabständen zwischen den ursprünglichen Preisen der Original-Biologika und ihren Nachahmerpräparaten zeigen sich für einzelne Wirkstoffe deutliche Unterschiede; zum Teil waren die Biosimilars sogar teurer als die Originale. Dies scheint auch mit der Zahl der Zweitanbieter zusammenzuhängen: Frühere Analysen des WIdO haben gezeigt, dass die Preisabstände umso größer sind, je mehr Anbieter im Markt vertreten sind (Schröder et al. 2019, 2020). Hieran zeigt sich, dass Wettbewerb selbst in so stark regulierten und technologisch anspruchsvollen Segmenten zu niedrigeren Preisen und Kosten führen kann. Allerdings fallen die Preise nach Patentablauf der Original-Biologika 


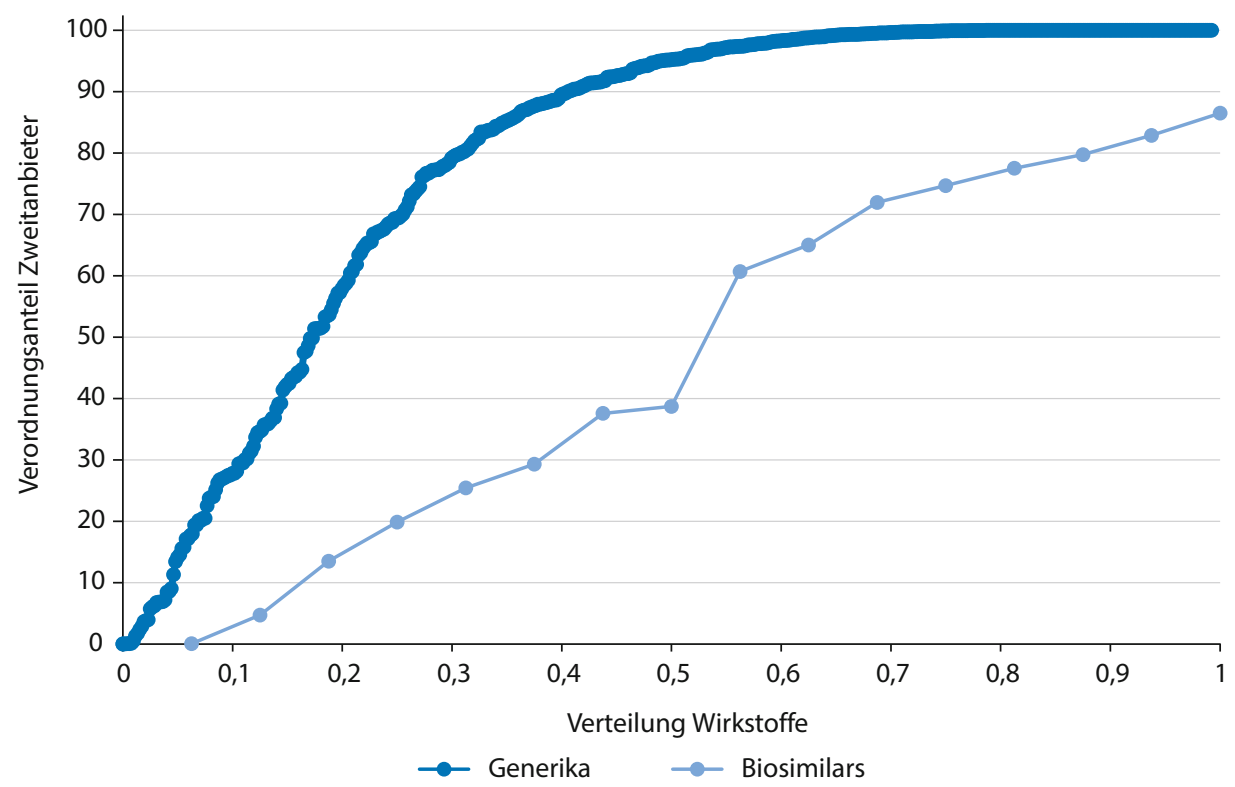

Arzneimittel-Kompass 2021

- Abb. 16.10 Zweitanbieteranteile im generikafähigen und im biosimilarfähigen Markt 2020 (Quelle: GKV-Arzneimittelindex)

bisher nicht auf das niedrige Niveau, das bei chemisch-synthetischen Generika üblich ist auch dies ist eine Folge der derzeit noch überschaubaren Anbietervielfalt (Schröder et al. 2019).

\section{- Rabattverträge}

Neben den für alle Krankenkassen gleich wirkenden Steuerungs- und Kostendämpfungsinstrumenten wie Festbeträgen oder den verschiedenen gesetzlichen Abschlägen (vgl. WIdO 2021) haben die Krankenkassen mit dem $\S 130$ a Absatz 8 SGB V seit 2003 die Möglichkeit, mit pharmazeutischen Herstellern individuelle Arzneimittelrabattverträge abzuschließen. Dabei schreiben die Krankenkassen auf der Ebene von einzelnen Wirkstoffen - gegebenenfalls eingeschränkt auf z. B. einzelne Darreichungsformen - öffentlich aus, welcher pharmazeutische Anbieter die Versorgung ihrer Versicherten mit diesem Wirkstoff für einen definierten Zeitraum übernehmen wird.
Mit dieser Anbieterfestlegung ist im Gegenzug ein finanzieller Rabatt für die Krankenkasse verbunden. Die Verträge wirken daher ausgabensenkend. Da die Wirkstoffauswahl selbstverständlich auch weiterhin der behandelnden Ärztin oder dem behandelnden Arzt obliegt, ist mit den Rabattverträgen keine Steuerung der Verordnungen auf bestimmte Wirkstoffe verbunden. Lediglich der Anbieter des verordneten Wirkstoffs wird über den Rabattvertrag bestimmt. In begründeten Ausnahmefällen kann jedoch von den Vorgaben der Rabattverträge abgewichen werden, beispielsweise wenn die individuelle ärztliche Therapieentscheidung begründet zugunsten eines bestimmten Anbieters ausfällt, in der Apotheke das abzugebende Arzneimittel nicht verfügbar ist oder zugunsten einer unverzüglichen Versorgung der Patient:innen entschieden wird. Im Jahr 2020 waren unter den insgesamt 2.488 ambulant verordneten Wirkstoffen und Wirkstoffkombinationen 650 bei mindestens 


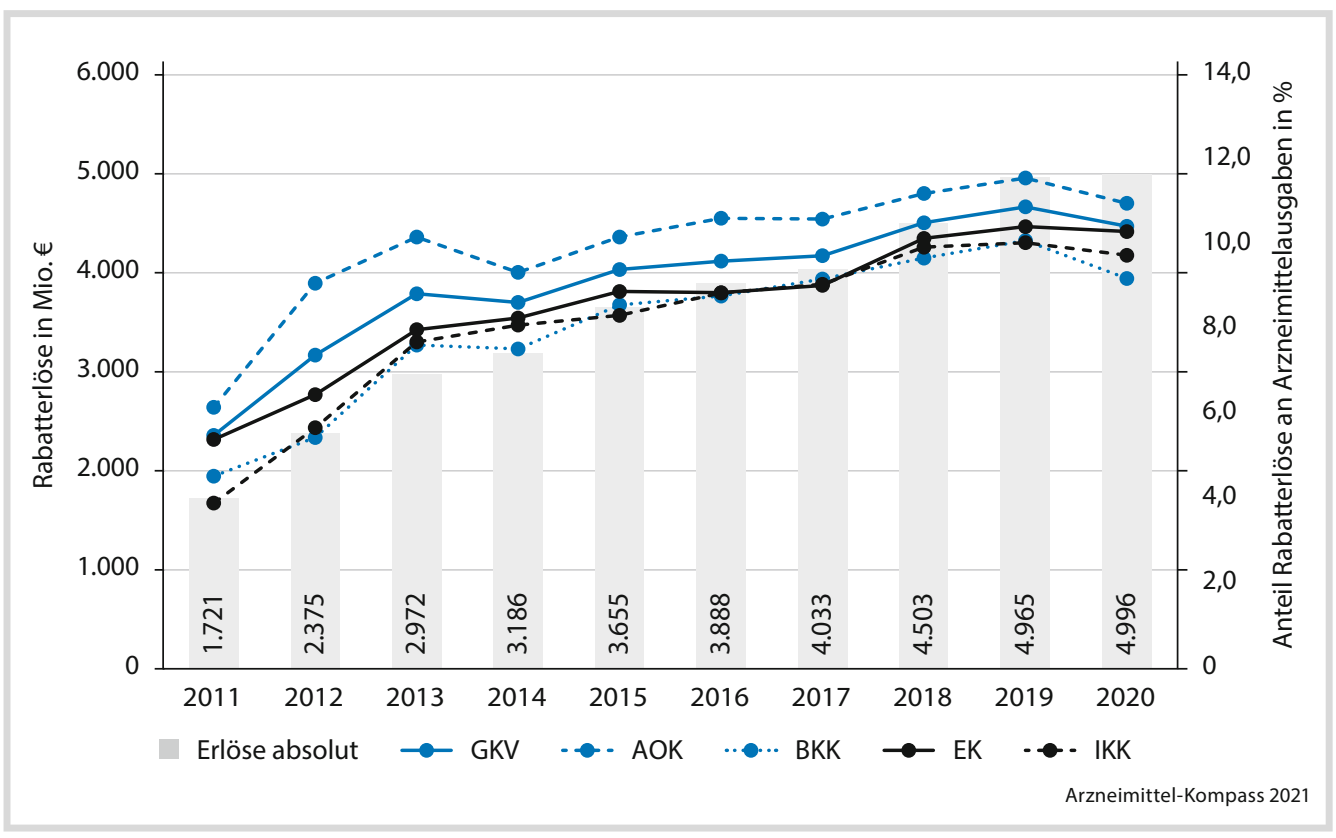

- Abb. 16.11 Erlöse aus Rabattverträgen seit 2011 nach amtlicher Statistik KJ1 (Quelle: WIdO nach amtlichen Statistiken des Bundesministeriums für Gesundheit (KJ1) für die jeweiligen Jahre)

einer Krankenkasse rabattiert. Im Wesentlichen beschränkt sich dies auf generika- bzw. biosimilarfähige Wirkstoffe, da vor allem hier der Anbieterwettbewerb genutzt werden kann.

\section{- Welche Einsparungen werden mit den} Rabattverträgen für die GKV erreicht?

Eine Darstellung der finanziellen Auswirkungen erlaubt das seit Mitte 2008 bestehende eigene Haushaltskonto der Krankenkassen in der amtlichen Statistik (KJ 1, seit 2010 ebenfalls in der vorläufigen Statistik KV 45), in dem die Einnahmen aus Rabattverträgen ausgewiesen werden. Für das Jahr 2008 wurde hier im zweiten Halbjahr erstmals ein Rabattbetrag von 310 Mio. $€$ gebucht. Für das Gesamtjahr 2020 beträgt nach der Finanzstatistik die GKV-Rabattsumme 5,00 Mrd. € (2019: 4,97 Mrd. €) und entspricht damit 10,4\% der Arzneimittelausgaben (- Abb. 16.11) bzw. 10,2\% des gesamten Bruttoumsatzes.

Dabei unterscheiden sich die Anteile der Rabatteinnahmen an den gesamten Arznei- mittelausgaben für die Kassenarten: Während die GKV insgesamt die Ausgaben zu $10,4 \%$ senken konnte, ist eine Spannweite von 9,2\% bei den Betriebskrankenkassen (BKK) bis zu $11,0 \%$ bei der AOK zu beobachten. Ähnlich verhalten sich auch die Ergebnisse je Versicherten der einzelnen Kassenarten (- Abb. 16.12): Auch hier liegen die Rabatterlöse bei den AOKs mit 71,95€ am höchsten, bei den BKKen mit $52,90 €$ je Versicherten am niedrigsten. Über alle Kassenarten betrachtet haben sich die Rabatterlöse seit 2011 von 24,71 auf $68,19 €$ je Versicherten nahezu verdreifacht.

\section{- Wie wirken sich Rabattverträge auf die Anbietervielfalt aus?}

Der Wettbewerb der Generikahersteller im Markt der GKV hat sich von Jahr zu Jahr durch neue generikafähige Wirkstoffe und neue Anbieter verstärkt. Ob diese Entwicklung, die durch sinkende Preise im Generikamarkt gekennzeichnet ist, auch mit einer Veränderung 


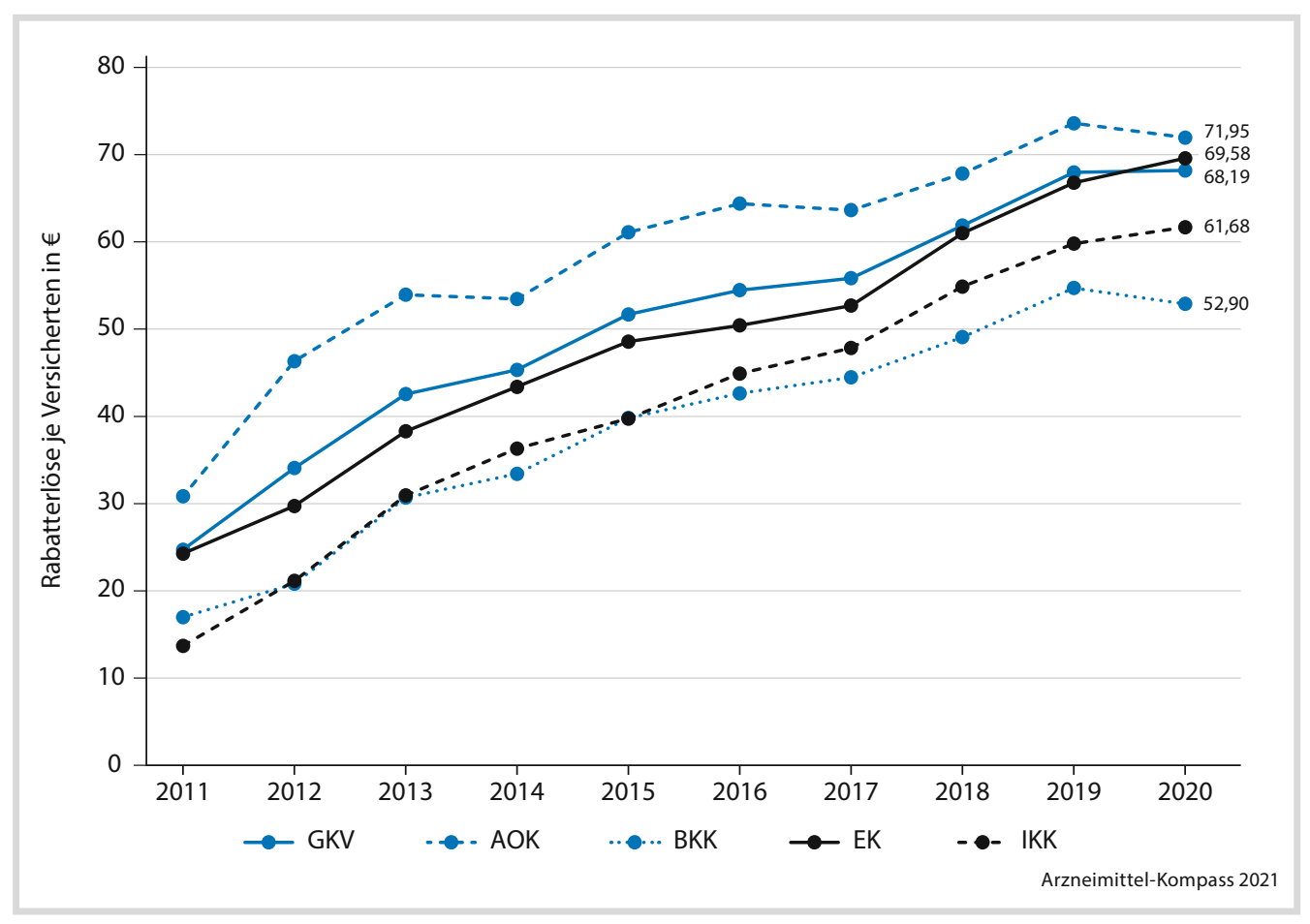

- Abb. 16.12 Rabatterlöse je Versicherten nach Kassenart seit 2011 nach amtlichen Statistiken KJ1 und KM6 (Quelle: WIdO nach amtlichen Statistiken des Bundesministeriums für Gesundheit (KJ1 und KM6) für die jeweiligen Jahre)

in der Anbieterstruktur einhergeht, wird im Folgenden thematisiert. Ein stärkerer Preisdruck kann zu einer stärkeren Konzentration der Absätze auf wenige Unternehmen führen, aus der sich möglicherweise eine stärkere Marktmacht oder auch eine Marktbereinigung ergibt. Mit einer größeren Zahl an im Wettbewerb stehenden Herstellern sinkt üblicherweise der Preis. Je größer das Marktvolumen ist, desto besser können die Hersteller ihre Fixkosten aufgrund der höheren Mengen verteilen.

Die Betrachtung der Umsatzkonzentration kann auf mehreren Ebenen erfolgen, beispielsweise für alle Arzneimittel, generikafähige Arzneimittel oder Arzneimittel einzelner Wirkstoffe. Im generikafähigen Markt kann davon ausgegangen werden, dass Generikaanbieter die Möglichkeit haben, sich mit entsprechenden Investitionen als Anbieter eines jeglichen Arzneimittels im patentfreien Markt zu betätigen. Die pharmazeutischen Unternehmer sind grundsätzlich in der Wahl der angebotenen Wirkstoffe frei und können ihr Wirkstoffportfolio flexibel und kurzfristig ändern. Die notwendigen Investitionen sind dabei vor allem im Vergleich zur Entwicklung innovativer Arzneimittel - relativ gering: Die Entwicklung eines Generikums erfordert mit 5 Mio. $€$ bei zwei Jahren Entwicklungszeit nur geringe finanzielle und zeitliche Aufwände (Bretthauer 2014). Generika-Anbieter können daher mit vergleichsweise wenig Aufwand flexibel ihr Marktsortiment verändern Daher handelt es sich hier um einen hochdynamischen Markt, was dafür spricht, die Marktkonzentration wirkstoffübergreifend $\mathrm{zu}$ messen. Im Folgenden wird daher die Umsatzkonzentration für den Gesamtmarkt und für den gesamten generikafähigen Markt betrachtet.

Zur Beurteilung der Stärke der Anbieterkonzentration in Märkten wird der Herfin- 
dahl-Hirschman-Index (HHI) verwendet, der unter anderem vom Statistischen Bundesamt und der Europäischen Kommission zur Beobachtung der Marktkonzentration herangezogen wird. Gemäß der Europäischen Kommission kennzeichnet ein Wert unterhalb von 1.000 eine niedrige Marktkonzentration, ein Wert bis 1.800 eine mittlere Konzentration und Werte oberhalb 1.800 kennzeichnen eine starke Marktkonzentration. Hier wird die Umsatzkonzentration im Jahr 2006, also vor Einführung der Rabattverträge, der Umsatzkonzentration im Markt im Jahr 2020 gegenübergestellt.

Im Ergebnis ist die Marktkonzentration im Generikamarkt, die bereits 2006 mit einem HHI von 478 insgesamt niedrig war, 2020 noch weiter auf 276 gesunken. Im Generikamarkt ist die Anbieterkonzentration ein wenig höher als im Gesamtmarkt (HHI: 219). Dies spiegelt wider, dass patentgeschützte Arzneimittel von vielen verschiedenen und nicht nur von einigen wenigen Anbietern entwickelt werden. Doch nicht nur an den eher abstrakten Werten des HHI lässt sich ein Rückgang der Anbieterkonzentration ablesen. Auch der Umsatzanteil, der sich auf die zehn oder 20 umsatzstärksten Anbieter konzentriert, ist seit 2006 kontinuierlich zurückgegangen. Im Generikamarkt vereinen beispielsweise die zehn stärksten Anbieter $43 \%$ der Umsätze auf sich, während es 2006 noch $53 \%$ waren (- Tab. 16.3).

Durch die heute üblichen transparenten, europaweiten Ausschreibungen haben auch kleinere und unbekanntere Hersteller eine bessere Möglichkeit, daran teilzunehmen. Da das Markenimage eines Anbieters für die Auswahl des Präparats keine Rolle mehr spielt, ermöglichen die Rabattvertragsausschreibungen diesen Herstellern größere Chancen, in den deutschen Markt einzutreten.

Ausführliche Analysen rund um die Rabattverträge, die das WIdO in den vergangenen Jahren durchgeführt hat, machen noch weitere positive Auswirkungen der Rabattverträge auf diesen Markt transparent. Durch Exklusivverträge lassen sich gegenüber Verträgen mit mehreren Anbietern höhere Einsparungen für die Krankenkassen erreichen. Exklusivverträge fördern zudem die Anbietervielfalt und bringen auch Vorteile für die Patient:innen in Form einer stabileren Versorgung. Darüber hinaus kann auch eine höhere Liefersicherheit erreicht werden (Schröder et al. 2020).

\section{- Festbeträge}

Arzneimittel, die bestimmte Merkmale teilen, können einer Festbetragsgruppe zugeordnet werden, mit der eine Erstattungshöchstgrenze einhergeht. Nach den Vorgaben des $\S 35$ SGB V bestimmt der Gemeinsame Bundesausschuss (G-BA), für welche Arzneimittelgruppen Festbeträge festgesetzt werden können. Im Einzelnen handelt es sich dabei um Arzneimittel mit entweder demselben Wirkstoff, pharmakologisch-therapeutisch vergleichbaren (insbesondere chemisch verwandten) Wirkstoffen oder therapeutisch vergleichbarer Wirkung (insbesondere Arzneimittelkombinationen). Festbeträge stellen damit eine indirekte Form der Preissteuerung dar, da sie nicht direkt in die Preissetzung der Hersteller eingreifen, sondern Erstattungshöchstgrenzen setzen. Die meisten Festbeträge werden im generikafähigen Markt festgesetzt.

Zum Stichtag 31. Dezember 2020 waren über das „GKV-Abrechnungsverzeichnis Arzneimittel" 39.974 einzelne im Handel befindliche Produkte in 5.031 Arzneimittel-Standardaggregaten mit 643 unterschiedlichen Wirkstoffen bzw. Wirkstoffkombinationen als Festbetrags-Arzneimittel gemeldet. Die 552,1 Mio. Verordnungen dieser Arzneimittel (80,7\% des Gesamtmarktes) verursachten im Jahr 2020 Nettokosten von zusammen 14,1 Mrd. $€(30,6 \%$ des Gesamtmarktes).

Im Bereich der Biosimilars wird dieses Instrument noch zurückhaltend genutzt. So gibt es aktuell bei fünf der 16 biosimilarfähigen Wirkstoffe eine Festbetragsgruppe (Enoxaparin, Erythropoietin, Etanercept, Filgrastim und Infliximab, Stand 31.12.2020). Im Jahr 2021 wurde ein wirkstoffübergreifender Festbetrag für die Gruppe der TNF-alpha-Hemmstoffe, unter anderem mit den verordnungsstarken 
- Tab. 16.3 Ausgewählte Kennwerte der Bruttoumsatzverteilung nach pharmazeutischen Anbietern in den Jahren 2006 und 2020

2006 $44 \%$ $63 \%$ 276

Herfindahl-Hirschman-Index ${ }^{1}$

\section{Generikamarkt}

Bruttoumsatzanteil der 10 umsatzstärksten Hersteller

Bruttoumsatzanteil der 20 umsatzstärksten Hersteller

Herfindahl-Hirschman-Index
2020 $35 \%$ $55 \%$

\begin{tabular}{|c|c|}
\hline $53 \%$ & $43 \%$ \\
\hline $69 \%$ & $60 \%$ \\
\hline 478 & 276 \\
\hline
\end{tabular}

*Der Herfindahl-Hirschman-Index ist die Summe der quadrierten Anteilswerte und kann Werte von 1 bis 10.000 annehmen, wobei der minimale Wert bei Gleichverteilung des Absatzes über alle Anbieter (= minimale Konzentration), der maximale Wert hingegen bei maximaler Konzentration (also wenn der gesamte Absatz auf einen einzigen Anbieter entfällt) erreicht wird. Der ausgewiesene Rückgang beim Herfindahl-Hirschman-Index zwischen 2006 und 2020 zeigt, dass die Marktkonzentration abgenommen hat.

Arzneimittel-Kompass 2021
Wirkstoffen Adalimumab und Etanercept sowie noch patentgeschützten Wirkstoffen festgesetzt, von dem eine deutliche Marktwirksamkeit erwartet wird. Festbeträge bereiten den Boden für (übergreifende) Preissenkungen auf Basis wettbewerbsfähiger Preise. Ein zusätzlicher Preiswettbewerb kann über Rabattverträge realisiert werden. Insofern kommt der 2019 mit dem Gesetz für mehr Sicherheit in der Arzneimittelversorgung (GSAV) beschlossenen und für die zweite Jahreshälfte 2022 erwarteten Liste des G-BA zur Austauschbarkeit von Biosimilars eine besondere Bedeutung zu.

\subsection{Wie verteilen sich die Nettokosten und Verordnungen auf die Pharmafirmen?}

Die Nettokosten im GKV-Arzneimittelmarkt 2020 in Höhe von 46,19 Mrd. $€$ entsprechen einem Umsatz der pharmazeutischen Industrie nach Herstellerabgabepreisen ohne Mehrwertsteuer von insgesamt 34,35 Mrd. $€$.

Die fünf Hersteller im gesamten Arzneimittelmarkt der GKV, auf die die höchsten Nettokosten im Jahr 2020 entfielen, vereinen einen Anteil von 26,3\% des Gesamtmarkts auf sich. In - Tab. 16.4 sind die 21 laut der Wirtschaftsprüfungsgesellschaft EY weltweit umsatzstärksten Pharmaunternehmen aufgeführt (EY 2021), sortiert nach GKV-Nettokosten. Bei diesen Unternehmen handelt es sich mit Ausnahme von Novartis eher um Hersteller, die das Gros ihrer Umsätze mit Pa- 
Tab. 16.4 Nettokosten, verordnete Tagesdosen und umsatzstärkstes Arzneimittel im GKV-Arzneimittelmarkt 2020 sowie EBIT-Margen der 21 Top-Pharmafirmen weltweit, sortiert nach GKV-Nettokosten

\begin{tabular}{|c|c|c|c|c|c|c|c|c|}
\hline $\begin{array}{l}\text { Rang } \\
\text { Netto- } \\
\text { kosten }\end{array}$ & Hersteller & Konzernsitz & $\begin{array}{l}\text { EBIT- } \\
\text { Margen* } \\
\text { in } \%\end{array}$ & $\begin{array}{l}\text { GKV } \\
\text { Netto- } \\
\text { kosten in } \\
\text { Mrd. } €\end{array}$ & $\begin{array}{l}\text { Kosten- } \\
\text { anteil } \\
\text { in } \%\end{array}$ & $\begin{array}{l}\text { DDD in } \\
\text { Mrd. }\end{array}$ & $\begin{array}{l}\text { Versor- } \\
\text { gungs- } \\
\text { anteil } \\
\text { in \% }\end{array}$ & $\begin{array}{l}\text { Umsatz- } \\
\text { stärkstes } \\
\text { Arznei- } \\
\text { mittel }\end{array}$ \\
\hline 1 & Novartis & Schweiz & 24,2 & 5,06 & 10,9 & 6,8 & 14,70 & Lucentis $^{\circledR}$ \\
\hline 2 & $\begin{array}{l}\text { Johnson \& } \\
\text { Johnson }\end{array}$ & USA & 24,2 & 2,16 & 4,7 & 0,1 & 0,14 & Zytiga $^{\circledR}$ \\
\hline 3 & Merck \& Co. & USA & 32,7 & 1,71 & 3,7 & 0,3 & 0,63 & Keytruda $^{\circledR}$ \\
\hline 4 & Roche & Schweiz & 33,4 & 1,63 & 3,5 & 0,0 & 0,07 & Avastin $^{\circledR}$ \\
\hline 5 & Sanofi & Frankreich & 20,4 & 1,59 & 3,4 & 1,4 & 2,99 & Lantus $^{\circledR}$ \\
\hline 6 & Pfizer** & USA & 24,2 & 1,49 & 3,2 & 0,3 & 0,75 & Ibrance $^{\circledR}$ \\
\hline 7 & $\begin{array}{l}\text { Bristol-Myers } \\
\text { Squibb** }\end{array}$ & USA & 18,2 & 1,14 & 2,5 & 0,2 & 0,35 & Eliquis $^{\circledR}$ \\
\hline 8 & Bayer & Deutschland & 16,5 & 1,06 & 2,3 & 0,4 & 0,77 & Xarelto $^{\circledR}$ \\
\hline 9 & Amgen & USA & 36,7 & 0,99 & 2,1 & 0,1 & 0,15 & Kanjinti $^{\circledR}$ \\
\hline 10 & Takeda & Japan & 8,9 & 0,95 & 2,1 & 0,1 & 0,24 & Entyvio $^{\circledR}$ \\
\hline 11 & Biogen & USA & 34,6 & 0,92 & 2,0 & 0,0 & 0,06 & Tecfidera $^{\circledR}$ \\
\hline 12 & Eli Lilly & USA & 30,8 & 0,85 & 1,8 & 0,3 & 0,55 & Trulicity $^{\circledR}$ \\
\hline 13 & Astra Zeneca & $\begin{array}{l}\text { Vereinigtes } \\
\text { Königreich }\end{array}$ & 15,6 & 0,83 & 1,8 & 0,3 & 0,59 & Symbicort $^{\circledR}$ \\
\hline 14 & AbbVie & USA & 34,3 & 0,79 & 1,7 & 0,1 & 0,23 & Humira $^{\circledR}$ \\
\hline 15 & $\begin{array}{l}\text { Boehringer } \\
\text { Ingelheim }\end{array}$ & Deutschland & 21,7 & 0,76 & 1,6 & 0,4 & 0,81 & Jardiance $^{\circledR}$ \\
\hline 16 & Novo Nordisk & Norwegen & 42,9 & 0,69 & 1,5 & 0,4 & 0,76 & Novorapid $^{\circledR}$ \\
\hline 17 & Astellas & Japan & 15,0 & 0,52 & 1,1 & 0,1 & 0,11 & Xtandi ${ }^{\circledR}$ \\
\hline 18 & GlaxoSmithKline & $\begin{array}{l}\text { Vereinigtes } \\
\text { Königreich }\end{array}$ & 24,2 & 0,46 & 1,0 & 0,3 & 0,68 & Viani $^{\circledR}$ \\
\hline 19 & Merck KGaA & Deutschland & 17,9 & 0,40 & 0,9 & 0,6 & 1,34 & Rebif $^{(\circledR)}$ \\
\hline 20 & Gilead Sciences & USA & 45,8 & 0,38 & 0,8 & 0,0 & 0,03 & Biktarvy $^{\circledR}$ \\
\hline \multirow[t]{3}{*}{21} & Otsuka & Japan & 14,9 & 0,08 & 0,2 & 0,0 & 0,01 & Abilify ${ }^{\circledR}$ \\
\hline & Top 21 & & 25,7 & 24,46 & 53,0 & 12,0 & 26,4 & \\
\hline & Gesamt & & & 46,19 & & 45,32 & & \\
\hline
\end{tabular}

*EBIT-Margen und weltweiter Pharma-Umsatz gemäß EY (2021)

**Marktdaten der Vertriebsgemeinschaft Pfizer/Bristol hälftig aufgeteilt

Quelle: GKV-Arzneimittelindex, teilweise basierend auf EY (2021)

Arzneimittel-Kompass 2021 
tentarzneimitteln erzielen: Zusammen ergeben sich $70 \%$ dieser Umsätze mit Patentarzneimitteln. Wie für den Patentmarkt typisch (siehe - Tab. 16.1), sind die Versorgungsanteile - gemessen in Tagesdosen - dagegen sehr gering. Die Patentarzneimittel dieser Hersteller tragen nur 4,4\% zur gesamten Versorgung nach Tagesdosen bei. So entstehen beispielsweise die Nettokosten von 2,2 Mrd. $€$ für Arzneimittel des Herstellers Johnson \& Johnson im Jahr 2020 zu $96 \%$ durch Patentarzneimittel. Allein die Arzneimittel dieses Herstellers sind damit für $4,7 \%$ der gesamten Nettokosten im Markt verantwortlich. Die damit verordneten etwa 66 Mio. Tagesdosen hingegen entsprechen einem Versorgungsanteil von gerade einmal $0,14 \%$ des Gesamtmarktes. Gemeinsam erreichen die Nettokosten aller 21 Unternehmen dieser Liste mit 24,46 Mrd. $€$ einen Anteil von $53 \%$ am Gesamtmarkt, nach Tagesdosen liegt der Anteil lediglich bei $26 \%$.

\section{- Gewinne der Arzneimittelhersteller}

Nach den bei fast allen großen Pharmakonzernen stark gestiegenen Gewinnen im Jahr 2019 konnten auch im Jahr 2020 nahezu alle wieder positive Ergebnisse vermelden. Zur Gruppe der fünf Spitzenreiter mit den höchsten EBITMargen $^{6}$ von jeweils deutlich über $30 \%$ gehören die Biotechnologie-Unternehmen Gilead, AbbVie, Biogen, Amgen und Novo Nordisk. Angeführt wird diese Rangliste vom Biotechnologie-Unternehmen Gilead mit einem Ergebnis von 45,8\%. Insgesamt lag der Anteil des EBIT am Gesamtumsatz bei den 21 TopUnternehmen stabil bei 25,7\% (EY 2021). Die Biotechnologie-Branche scheint dabei insgesamt nach wie vor besonders lukrativ zu sein: Im Vergleich mit durchschnittlichen EBITMargen der Top-Pharmaunternehmen ohne biotechnologischen Schwerpunkt von 21,0\% lagen deren Margen im Schnitt mit 36,7\%

6 Earnings Before Interest and Taxes; Gewinn vor Zinsen und Steuern. deutlich höher (EY 2021). Die absolut höchsten Pharma-Gewinne konnte Roche weltweit mit 14,5 Mrd. $€$ erwirtschaften. Insgesamt wird der internationale Pharmamarkt von USKonzernen dominiert. Die deutschen Konzerne Bayer und Boehringer rangieren weltweit nach Umsatz erst auf den Plätzen 15 und 17 (EY 2021). Für den deutschen Arzneimittelmarkt können sich zumindest Bayer und weitere europäische Konzerne weiter vorne behaupten, dennoch sind auch im deutschen Markt die US-Konzerne stark vertreten und erzielen in dieser Übersicht zusammen ca. $43 \%$ der Nettokosten.

Gesamtwirtschaftlich betrachtet werden in der Branche Pharma und Biotechnologie sowohl in Europa als auch in den Vereinigten Staaten die mit Abstand höchsten EBIT-Margen erzielt. Im Vergleich zur Pharmabranche lag diese Kennzahl für das Jahr 2018 in der ebenfalls finanzkräftigen Branche der Informationstechnologie für die besonders in Forschung und Entwicklung starken Unternehmen lediglich bei $18,8 \%$ (EY 2019). Auch hier gibt es mit Apple, Microsoft und Google durchaus erfolgreiche Unternehmen, die aber in ihren EBIT-Margen bei weitem nicht an die der oben genannten Biotechnologieunternehmen herankommen.

Diese Beobachtung wird auch durch die Studie von Ledley et al. (2020) unterstützt, die zeigen konnten, dass die Rentabilität der großen pharmazeutischen Unternehmen in den Jahren 2000 bis 2018 signifikant höher war als die von großen Unternehmen aus anderen Branchen: So lag die durchschnittliche internationale EBITDA-Marge ${ }^{7}$ der untersuchten pharmazeutischen Unternehmen bei $29,4 \%$, während sie bei den betrachteten Großunternehmen aus anderen Branchen bei 19,0\% lag.

7 Earnings Before Interest, Taxes, Depreciation and Amortization; Gewinn vor Zinsen, Steuern, Abschreibungen auf Sachanlagen und Abschreibungen auf immaterielle Vermögensgegenstände. 


\subsection{Der Arzneimittelmarkt in der COVID-19-Pandemie}

Auch im Arzneimittelmarkt der GKV sind die Spuren der globalen COVID-19-Pandemie deutlich erkennbar. Wie in den vorangegangenen Abschnitten gezeigt, sind sowohl die Gesamtumsätze wie auch die Menge an insgesamt verbrauchten Tagesdosen und nicht zuletzt auch die Gewinne der großen Pharmaunternehmen weiterhin deutlich angestiegen; die Branche scheint also insgesamt betrachtet deutlich weniger von Einschnitten betroffen gewesen zu sein als andere Branchen (siehe - Kap. 7 von Kirchhoff zur Krisenresistenz der Pharmabranche). Zu den Verbräuchen und Umsätzen der Impfstoffe gegen COVID-19 lassen sich für Deutschland keine Aussagen treffen, da diese zentral über das Bundesgesundheitsministerium beschafft und verteilt wurden und in den verfügbaren Daten nicht enthalten sind. Im Folgenden werden einige Besonderheiten im Markt 2020 näher betrachtet.

Üblicherweise gibt es von Monat zu Monat in jedem Jahr Schwankungen im Arzneimittelverbrauch, da sich unter anderem die Lage beweglicher Feiertage oder der Haupturlaubszeiten von Jahr zu Jahr unterscheiden. Ebenso können Zeitraum und Intensität saisonaler Krankheitsereignisse wie beispielsweise Grippe- oder Erkältungswellen einen Einfluss auf die monatlichen Gesamtverbräuche haben. Im Jahr 2020 zeigt sich hingegen eine durchaus große Abweichung des monatlichen Arzneimittelverbrauchs, insbesondere im Monat März, in dem im Vergleich mit den durchschnittlichen Vorjahreswerten über $15 \%$ mehr Packungen verordnet wurden. In den folgenden Monaten April und Mai hingegen lag die Zahl der Packungen zwischen 8 und $11 \%$

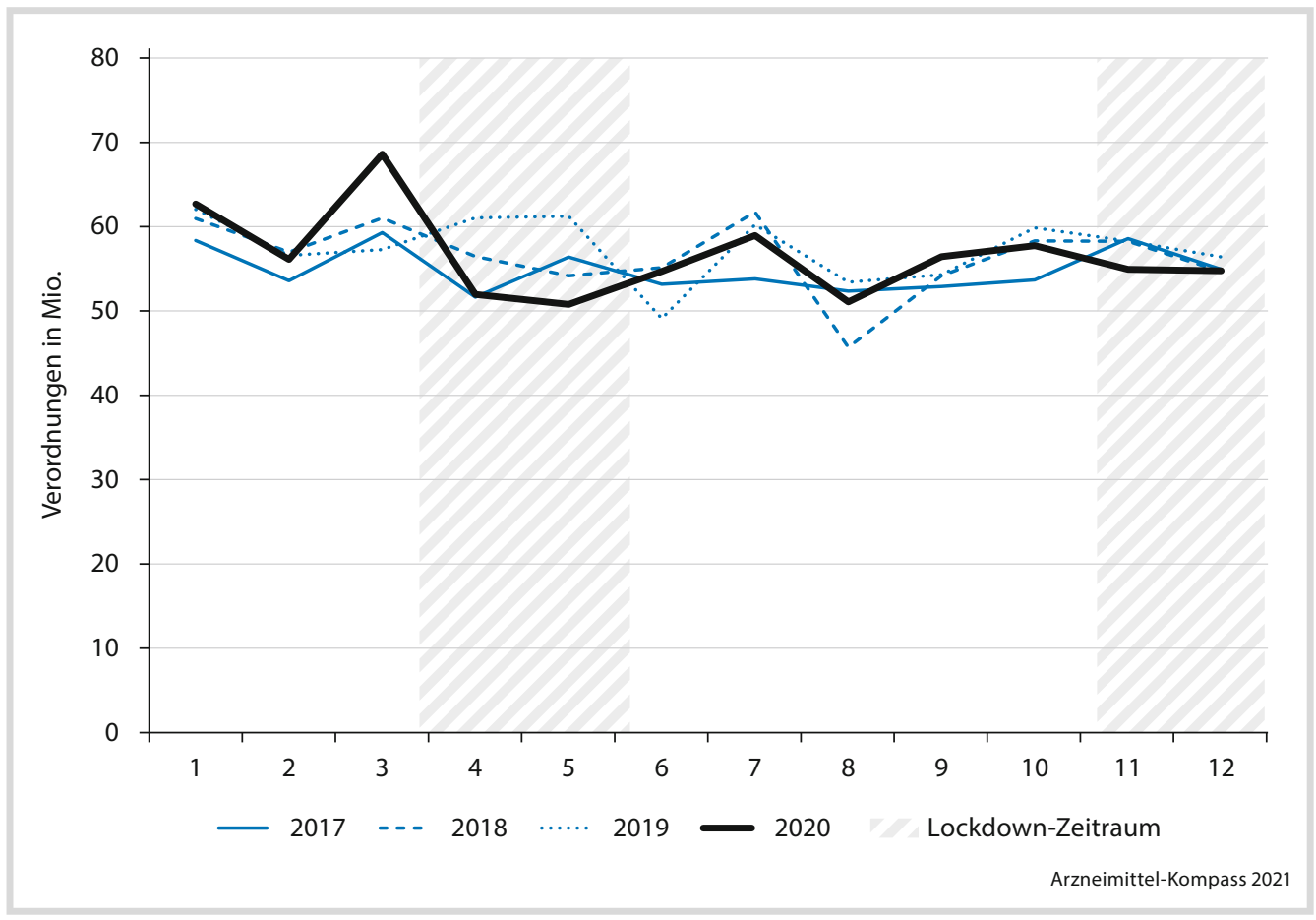

- Abb. 16.13 Monatliche Verordnungsmengen in den Jahren 2017 bis 2020; Zeiträume der bundesweiten LockdownMaßnahmen 2020 hervorgehoben (Quelle: GKV-Arzneimittelindex) 


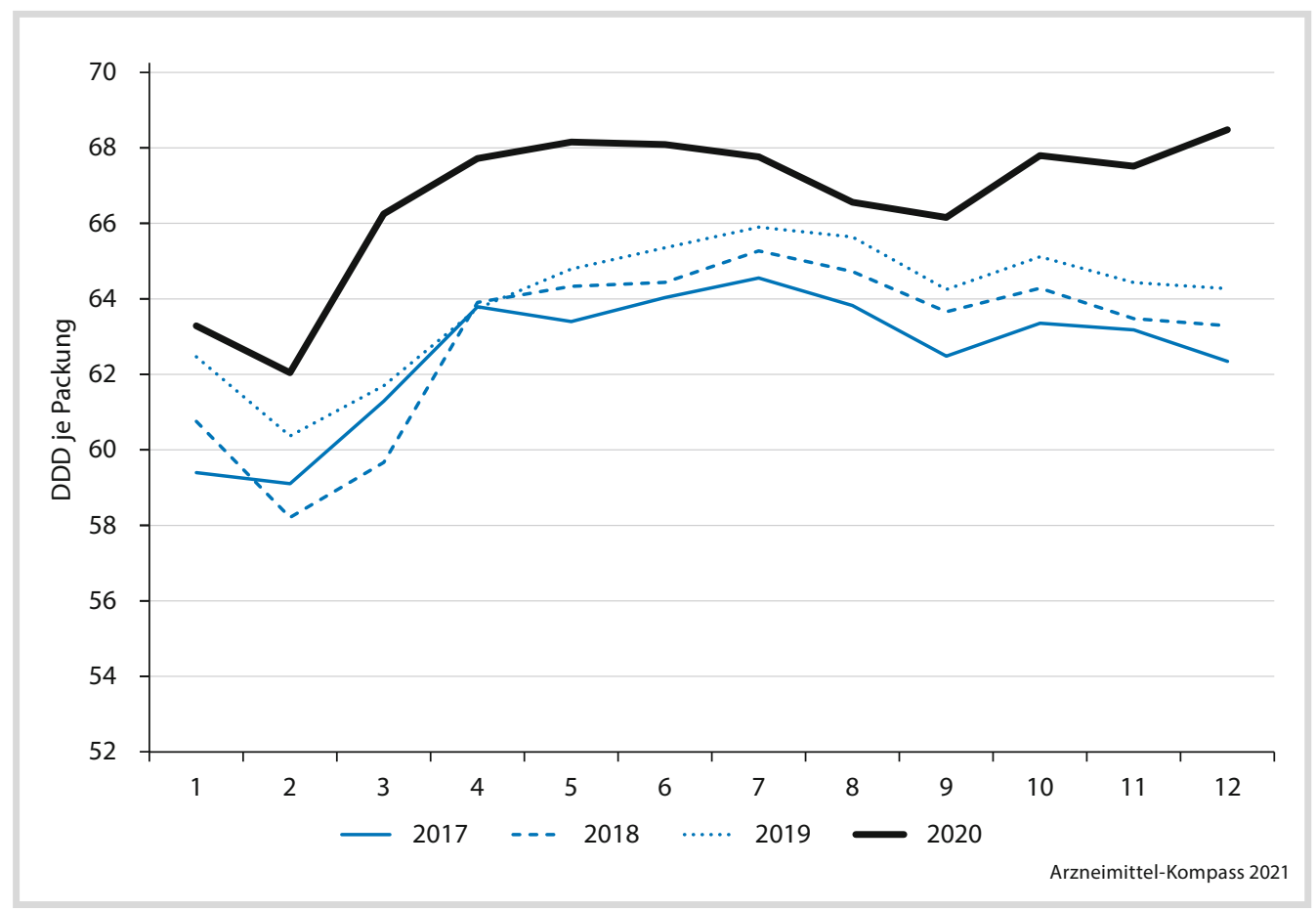

- Abb. 16.14 Monatlich durchschnittlich verordnete Packungsgrößen nach Tagesdosen je Packung in den Jahren 2017 bis 2020 (zur Verdeutlichung ist die y-Achse gestaucht dargestellt) (Quelle: GKV-Arzneimittelindex)

unter den durchschnittlichen Monatswerten. Auch in den Monaten November und Dezember wurden etwas weniger Arzneimittelrezepte in den Apotheken eingelöst als üblicherweise (•Abb. 16.13). Für diese ungewöhnliche Entwicklung kann ein Zusammenhang mit den bundesweiten Lockdown-Maßnahmen mit Ausgangsbeschränkungen zur Kontaktvermeidung während der COVID-19-Pandemie angenommen werden, die von Mitte März bis Mai sowie schrittweise ab November ergriffen wurden. In dieser Zeit blieben viele Arztbesuche aus oder wurden verschoben (Mangiapane et al. 2021). Der ungewöhnlich hohe Verbrauch im März lässt sich als Vorzieheffekt interpretieren: Viele Versicherte haben sich angesichts der bevorstehenden Maßnahmen rechtzeitig mit ihren Dauermedikationen versorgt. Vergleichbar auffällig lässt sich dies allerdings nicht für die zweite Lockdownphase zum Jahresende beobachten, wofür die Erfah- rungen aus dem ersten Lockdown ein Grund sein können.

Trotz der dadurch insgesamt über das gesamte Jahr 2020 um 1,6\% gesunkenen Menge an verordneten Arzneimittelpackungen hat der Verbrauch an Tagesdosen gemessen um 2,4\% zugenommen. Dies lässt sich über eine stärkere Zunahme von größeren Packungen mit mehr enthaltenen Tagesdosen erklären, wie anhand der in $\bullet$ Abb. 16.14 dargestellten monatlich durchschnittlich verordneten Packungsgröße erkennbar ist. So lag insbesondere in den Phasen der Kontakteinschränkungen im März und April sowie November und Dezember die durchschnittliche Packungsgröße um ca. 6 bis $9 \%$ über den durchschnittlichen Werten der Vorjahre. Ob sich hieraus eine dauerhafte Entwicklung zu deutlich größeren Packungen ergibt, wird sich erst in den kommenden Jahren bei Normalisierung der Voraussetzungen beurteilen lassen. 


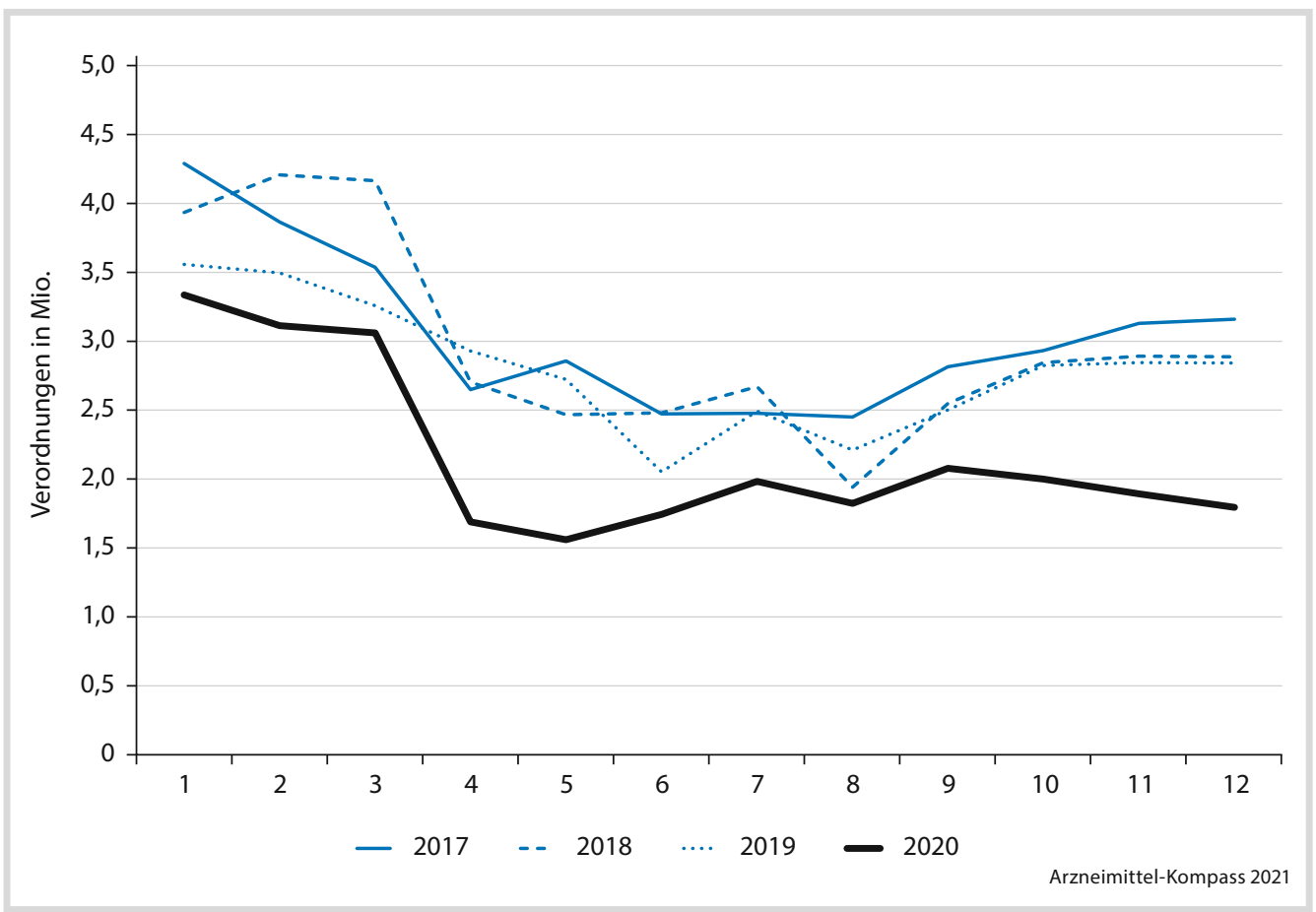

- Abb. 16.15 Monatliche Verordnungsmengen von Antibiotika (ATC-Gruppe J01) in den Jahren 2017 bis 2020 (Quelle: GKV-Arzneimittelindex)

Insgesamt lässt sich vor allem für chronische Erkrankungen mit dauerhaft und regelmäßig einzunehmender Medikation annehmen, dass den GKV-Versicherten eine weitgehend kontinuierliche Versorgung mit Arzneimitteln möglich war. Die Untersuchung des WIdO für den März 2020 zeigt, dass insbesondere bei den dauerhaft von einer großen Zahl an Patient:innen eingenommenen HerzKreislauf-, Magen-Darm- und Diabetes-Medikamenten höhere Mengen an Tagesdosen als üblich in Anspruch genommen wurden (Schröder et al. 2020).

Für einzelne Indikationsbereiche lassen sich auf der anderen Seite aber auch gegensätzliche Entwicklungen im Jahr 2020 beobachten. So sind insbesondere die Antibiotikaverordnungen im Vergleich zu den Vorjahren zum Teil erheblich zurückgegangen. Auch für die in - Abb. 16.15 dargestellten Vorjahre lassen sich zwar insbesondere in den „klassischen“
Erkältungsmonaten Dezember bis März Unterschiede erkennen, abhängig von der jeweiligen Intensität von Erkältungswellen. Jedoch lagen die monatlichen Verordnungsmengen im Jahr 2020 durchweg deutlich unter den üblicherweise monatlich verordneten Packungsmengen, im Mittel um 27\%, in der Spitze in den Lockdown-Monaten April, Mai und Dezember 2020 sogar um ca. $40 \%$ darunter (- Abb. 16.15). Ähnliches zeigt sich auch für die Husten- und Erkältungsmittel, die in der Regel nur für Kinder und Jugendliche erstattungsfähig sind. Hier betragen die Rückgänge im Vergleich zu den durchschnittlichen Vorjahreswerten in den genannten Monaten sogar 55 bis $64 \%$.

Für diese deutlichen Rückgänge ist ein Zusammenhang mit den Kontaktbeschränkungen sowohl für private als auch berufliche und schulische Interaktionen anzunehmen. Wo Menschen sich nicht begegnet sind, wurden 


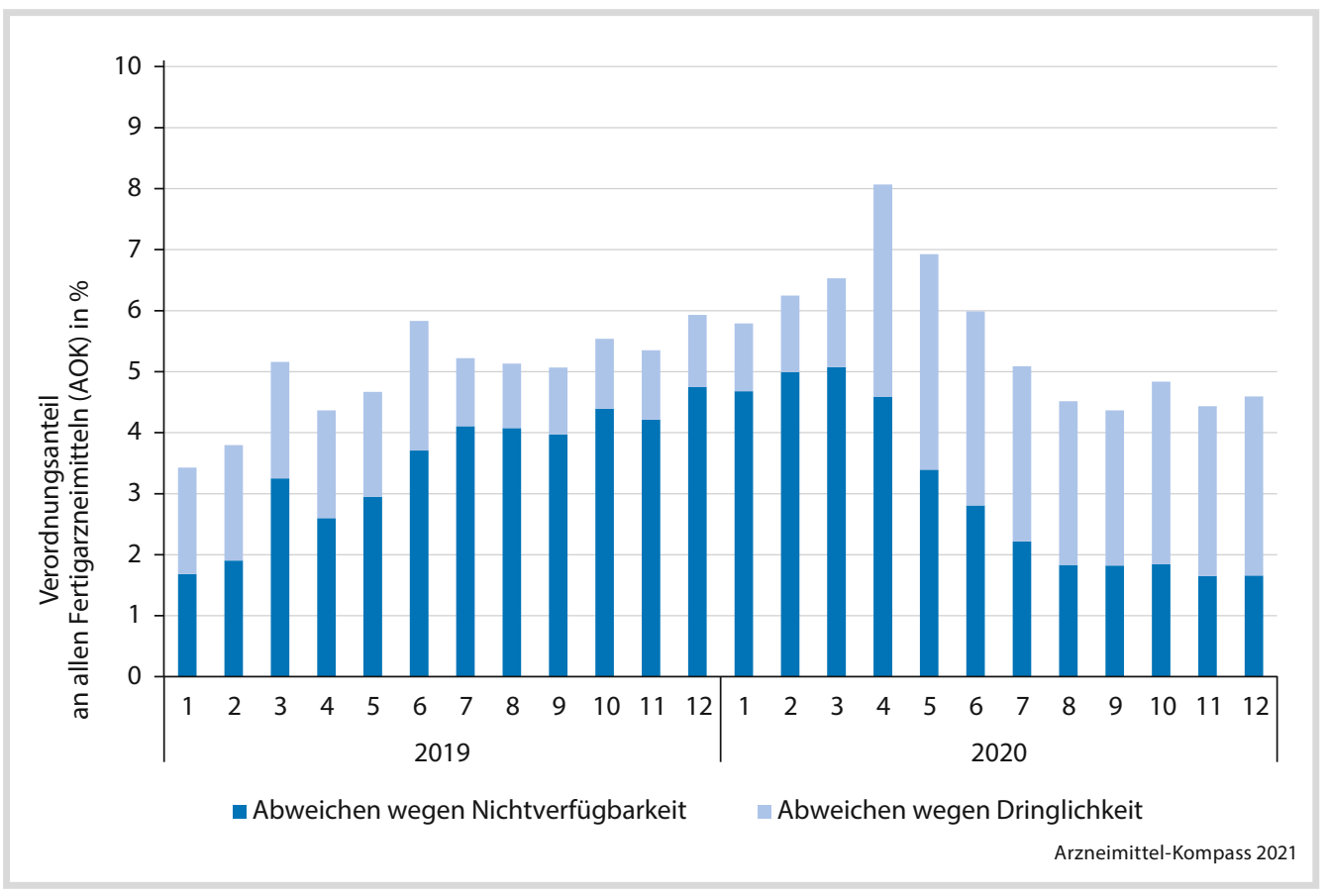

- Abb. 16.16 Monatliche Anteile der Fertigarzneimittel, für die Apotheken eine Ersatzabgabe wegen Nichtverfügbarkeit oder Dringlichkeit vorgenommen haben (Daten für AOK-Versicherte) (Quelle: GKV-Arzneimittelindex)

nicht nur die mit den Maßnahmen beabsichtigten Übertragungen des Coronavirus vermieden, sondern es ist zu vermuten, dass auch Infektionen mit anderen Erregern deutlich seltener stattgefunden haben und infolgedessen diese mit weniger Arzneimitteln behandelt wurden. Auch die Beachtung der sogenannten AHA+A-Regel (Abstand halten, Hygieneregeln beachten, Alltagsmasken tragen, CoronaWarn-App aktivieren (BMG 2020)) wird hier einen Einfluss gehabt haben. Es kann jedoch auch angenommen werden, dass selbst bei erlebten Infekten seltener eine Arzneimitteltherapie durchgeführt wurde, um Kontakte in Arztpraxen und Apotheken zu vermeiden.

Verschiedentlich wurde eine Verschlechterung hinsichtlich der Arzneimittelversorgung der Versicherten in Bezug auf die Versorgungskontinuität und Liefersicherheit befürchtet (Reinhardt 2020; Süddeutsche Zeitung 2020). So wurde angesichts der LockdownMaßnahmen auch in anderen Teilen der Welt
- insbesondere Indien und China - mit Fabrikschließungen und Handelsbeschränkungen befürchtet, dass es zu einer Verknappung an Arzneimitteln kommen könnte. Die Auswertung der in den Apotheken durchgeführten Ersatzabgaben $^{8}$ lässt jedoch keine Zunahme an nicht verfügbaren Arzneimitteln erkennen: Die Ersatzabgabe wegen Nichtverfügbarkeit ist sogar von durchschnittlich 3,4\% der Verordnungen 2019 auf 3,1\% im Jahr 2020 zurückgegangen.

Die Apotheken hatten zur Vermeidung zusätzlicher Kontakte während der Pandemiezeit die Möglichkeit, auch ohne Nachweis einer

8 Grundsätzlich sind Apotheken verpflichtet, statt des namentlich verordneten Arzneimittels ein wirkstoffgleiches vergleichbares Arzneimittel abzugeben, wenn beispielsweise Festbeträge oder Rabattverträge der Krankenkassen dies vorsehen. In begründeten Ausnahmefällen kann jedoch hiervon abgewichen werden, bspw. wenn das Arzneimittel nicht verfügbar ist oder zugunsten einer unverzüglichen Versorgung der Patient:innen entschieden wird. 
Nichtverfügbarkeit ein vergleichbares, wirkstoffidentisches Arzneimittel abzugeben. Diese mit „Abweichen wegen Dringlichkeit“ bezeichneten Ersatzabgaben haben ab April 2020 leicht zugenommen, für das Jahr 2020 auf durchschnittlich 2,5\% der Fertigarzneimittel gegenüber $1,5 \%$ im Vorjahr (• Abb. 16.16). Die gemeinsame Betrachtung dieser Ersatzabgaben lässt jedoch keine übermäßige Zunahme der Nichtverfügbarkeiten in den Zeiten der Pandemie erkennen; in allen dokumentierten Fällen wurde vielmehr eine alternative Versorgung der Versicherten mit gleichwertigen Arzneimitteln in den Apotheken sichergestellt.

Wie für viele Lebensbereiche wird es auch für den Arzneimittelmarkt interessant sein zu beobachten, welche offenbar pandemiebedingten Trends erhalten werden und welche sich gegebenenfalls wieder aufheben oder gar ins Gegenteil umschlagen.

\section{Literatur}

Bretthauer B (2014) Biosimilars 2.0. Weichen für die nachhaltige Versorgung stellen. IMPLICONplus 06

Bundesministerium für Gesundheit (2016) Arzneimittelmarktneuordnungsgesetz (AMNOG). https://www. bundesgesundheitsministerium.de/service/begriffevon-a-z/a/arzneimittelmarktneuordnungsgesetzamnog.html. Zugegriffen: 3. Juli 2020

Bundesministerium für Gesundheit (2020) Mit der AHA+A-Formel gegen Corona. https://www. bundesregierung.de/breg-de/themen/coronavirus/ aha-a-formel-1774474. Zugegriffen: 7. Juli 2021

Bundesministerium für Gesundheit (2021) Gesetzliche Krankenversicherung Vorläufige Rechnungsergebnisse 1. bis 4. Quartal 2019. https://www. bundesgesundheitsministerium.de/fileadmin/Dateien/ 3_Downloads/G/GKV/210302_PM_Anlage_ barrierefrei_1.-4._Qu._2020_bf_Tabelle.pdf. Zugegriffen: 23. Juni 2021

EY (2019) Top 500 F\&E: Wer investiert am meisten in Innovationen? https://assets.ey.com/content/dam/ ey-sites/ey-com/de_de/news/2019/07/ey-top-500-feunternehmen-der-welt-2019.pdf?download. Zugegriffen: 5. Juli 2021

EvaluatePharma (2018) Orphan Drug Report 2018. http://www.evaluategroup.com/public/Reports/ EvaluatePharma-Orphan-Drug-Report-2018.aspx.
EvaluatePharma (2020) Orphan Drug Report 2020. https:// www.evaluate.com/orphan-drugs. Zugegriffen: 5. Juli 2021

EY (2021) Die größten Pharmafirmen weltweit. Analyse der wichtigsten Finanzkennzahlen der Geschäftsjahre 2018, 2019 und 2020. https://assets.ey.com/ content/dam/ey-sites/ey-com/de_de/news/2021/06/ ey-pharma-bilanzen-2021.pdf?download. Zugegriffen: 5. Juli 2021

Ledley FD, McCoy SS, Vaughan G et al (2020) Profitability of large pharmaceutical companies compared with other large public companies. JAMA 323(9):834-843

Litsch M, Bogum J, Hopfgarten C (2019) Anpassungsbedarf in der Arzneimittelversorgung. G\&s Gesundheits- Sozialpolitik 73(2):13-18

Ludwig WD (2019) Orphan Drugs aus Sicht der Arzneimittelkommission der deutschen Ärzteschaft. Chancen und Herausforderungen. Internist 60(4):399-404

Mangiapane S, Zhu L, Kretschmann J et al (2021) Veränderung der vertragsärztlichen Leistungsinanspruchnahme während der COVID-Krise, Berlin 16.04.2021. https://www.zi.de/fileadmin/ images/content/Publikationen/Trendreport_4_ Leistungsinanspruchnahme_COVID_2021-04-19. pdf. Zugegriffen: 6. Juli 2021

Marselis D, Hordijk L (2020) From blockbuster to „nichebuster“: how a flawed legislation helped create a new profit model for the drug industry. BMJ 370(2020):m2983. https://doi.org/10.1136/bmj. m2983

Reichelt H (1988) Eine Methode der statistischen Komponentenzerlegung. Konzept einer erweiterten Index-Analyse volkswirtschaftlicher Änderungsraten. https://www.wido.de/fileadmin/Dateien/Dokumente/ Publikationsdatenbank/wido_all_mat31_1988_0109. pdf (WIdO-Materialien 31, Bonn)

Reinhardt K (2020) „Die Lieferengpässe bei Impfstoffen beunruhigen mich sehr". Interview im Tagesspiegel vom 26.08.2020. https://www.tagesspiegel. de/wirtschaft/aerztepraesident-klaus-reinhardt-iminterview-die-lieferengpaesse-bei-impfstoffenbeunruhigen-mich-sehr/26129292.html. Zugegriffen: 7. Juli 2021

Schröder M, Telschow C (2017) Der GKVArzneimittelmarkt 2016: Trends und Marktsegmente. In: Schwabe U et al (Hrsg) Arzneiverordnungs-Report 2017. Springer, Berlin, S 137-166

Schröder M, Lohmüller J, Telschow C (2019) Kostenentlastung durch Biosimilars - Was ist real, was wäre möglich? Kompendium Biosimilars 4:32-38. https:// doi.org/10.1055/a-0755-1936

Schröder M, Lohmüller J, Telschow C et al (2020) Der GKV-Arzneimittelmarkt. Bericht 2020. Wissenschaftliches Institut der AOK, Berlin. https:// www.wido.de/forschung-projekte/arzneimittel/gkvarzneimittelmarkt/.Zugegriffen: 8. Juli 2021 
Schweitzer SO, Lu ZJ (2018) Pharmaceutical economics and policy: perspectives, promises, and problems. Oxford University Press

Süddeutsche Zeitung (2020) Lieferengpässe für Medikamente möglich. Online Meldung aus dem dpa Newskanal vom 07.09.2020. https:// www.sueddeutsche.de/gesundheit/gesundheit- lieferengpaesse-fuer-medikamente-moeglichdpa.urn-newsml-dpa-com-20090101-200907-99459572. Zugegriffen: 7. Juli 2021

WIdO (2021) Der GKV-Arzneimittelmarkt: Klassifikation, Methodik und Ergebnisse 2021. https://wido.de/ forschung-projekte/arzneimittel/methoden/?L=0

Open Access Dieses Kapitel wird unter der Creative Commons Namensnennung 4.0 International Lizenz (http:// creativecommons.org/licenses/by/4.0/deed.de) veröffentlicht, welche die Nutzung, Vervielfältigung, Bearbeitung, Verbreitung und Wiedergabe in jeglichem Medium und Format erlaubt, sofern Sie den/die ursprünglichen Autor(en) und die Quelle ordnungsgemäß nennen, einen Link zur Creative Commons Lizenz beifügen und angeben, ob Änderungen vorgenommen wurden.

Die in diesem Kapitel enthaltenen Bilder und sonstiges Drittmaterial unterliegen ebenfalls der genannten Creative Commons Lizenz, sofern sich aus der Abbildungslegende nichts anderes ergibt. Sofern das betreffende Material nicht unter der genannten Creative Commons Lizenz steht und die betreffende Handlung nicht nach gesetzlichen Vorschriften erlaubt ist, ist für die oben auf geführten Weiterverwendungen des Materials die Einwilligung des jeweiligen Rechteinhabers einzuholen.

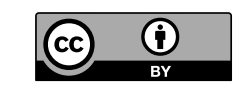

